\title{
A HIGHER ORDER ACCURATE FINITE ELEMENT METHOD FOR VISCOUS COMPRESSIBLE FLOWS
}

\author{
by \\ Daryl Lawrence Bonhaus \\ Dissertation submitted to the Faculty of the \\ Virginia Polytechnic Institute and State University \\ in partial fulfillment of the requirements for the degree of \\ DOCTOR OF PHILOSOPHY \\ in
}

Aerospace and Ocean Engineering

APPROVED:

Bernard Grossman, Chairman

W. Kyle Anderson

Robert Walters
Joseph Schetz

William Mason

November, 1998

Blacksburg, VA

This research was conducted at NASA LaRC. 


\title{
A HIGHER ORDER ACCURATE FINITE ELEMENT METHOD FOR VISCOUS COMPRESSIBLE FLOWS
}

\author{
by \\ Daryl L. Bonhaus \\ Committee Chairman: Bernard Grossman \\ Aerospace and Ocean Engineering
}

(ABSTRACT)

The Streamline Upwind/Petrov-Galerkin (SU/PG) method is applied to higher-order finite-element discretizations of the Euler equations in one dimension and the NavierStokes equations in two dimensions. The unknown flow quantities are discretized on meshes of triangular elements using triangular Bezier patches. The nonlinear residual equations are solved using an approximate Newton method with a pseudotime term. The resulting linear system is solved using the Generalized Minimum Residual algorithm with block diagonal preconditioning.

The exact solutions of Ringleb flow and Couette flow are used to quantitatively establish the spatial convergence rate of each discretization. Examples of inviscid flows including subsonic flow past a parabolic bump on a wall and subsonic and transonic flows past a NACA 0012 airfoil and laminar flows including flow past a a flat plate and flow past a NACA 0012 airfoil are included to qualitatively evaluate the accuracy of the discretizations. The scheme achieves higher order accuracy without modification. Based on the test cases presented, significant improvement of the solution can be expected using the higherorder schemes with little or no increase in computational requirements. The nonlinear system also converges at a higher rate as the order of accuracy is increased for the same number of degrees of freedom; however, the linear system becomes more difficult to solve. Several avenues of future research based on the results of the study are identified, including improvement of the SU/PG formulation, development of more general grid generation strategies for higher order elements, the addition of a turbulence model to extend the method to high Reynolds number flows, and extension of the method to three-dimensional flows. An appendix is included in which the method is applied to inviscid flows in three 
dimensions. The three-dimensional results are preliminary but consistent with the findings based on the two-dimensional scheme. 


\section{Acknowledgments}

I am grateful for the assistance of Dr. Mark H. Carpenter for valuable technical discussions and a review of this manuscript. I am also grateful to Christian Whiting for helpful discussions during his visits to the Langley Research Center and to Dr. Thomas W. Roberts for many helpful discussions.

I would particularly like to thank Dr. W. Kyle Anderson, who has been both a friend and a mentor to me throughout my career. 


\section{Table of Contents}

Abstract

Acknowledgments $\quad$ iv

Table of Contents $\quad$ v

List of Figures $\quad$ vii

Chapter 1 Introduction $\quad 1$

Chapter 2 The Streamline Upwind/Petrov-Galerkin Method 6

2.1 Scalar Advection - One Dimension .......................................................... 6

2.2 Advective Systems - One Dimension ..........................................................

2.3 Scalar Advection - Multiple Dimensions ...................................................

2.4 Advective Systems - Multiple Dimensions ................................................. 10

Chapter 3 Quasi-One-Dimensional Euler Equations 11

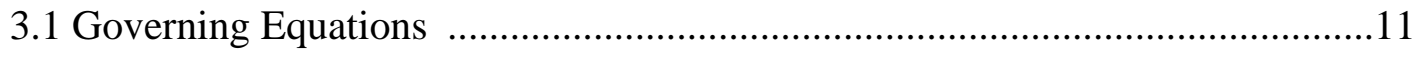

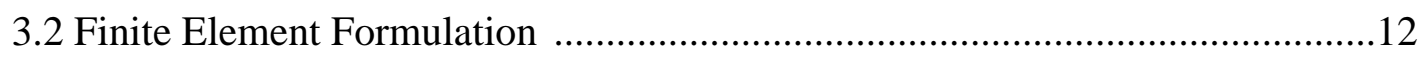

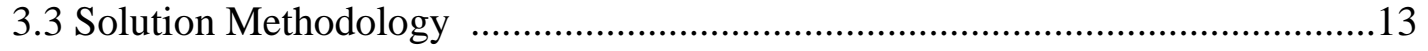

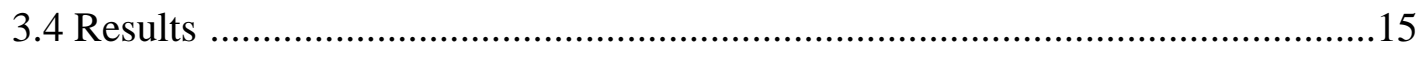

Chapter 4 Two-Dimensional Navier-Stokes Equations 22

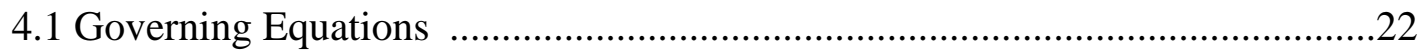

4.2 Finite Element Formulation ..................................................................25 


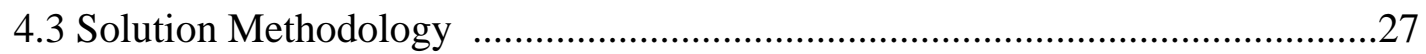

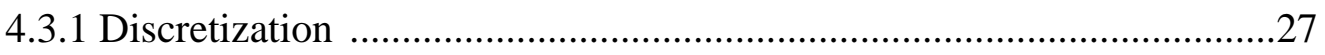

4.3.2 Solution Procedure …………………..................................................

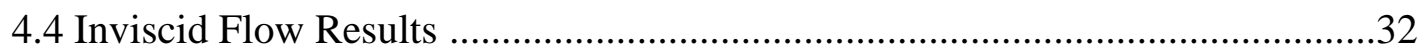

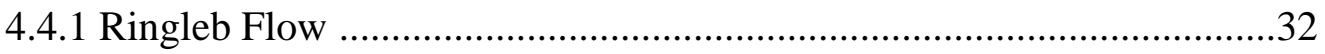

4.4.2 Bump on a Wall ……………………………......................................

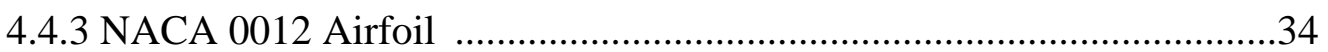

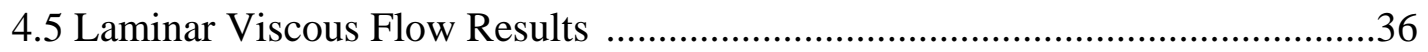

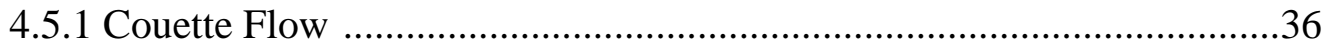

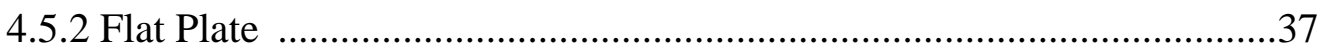

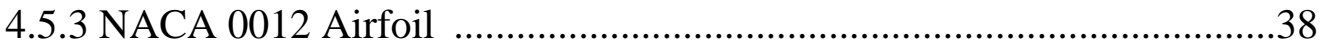

Chapter 5 Concluding Remarks

References $\quad 62$

Appendix A Three-Dimensional Euler Equations

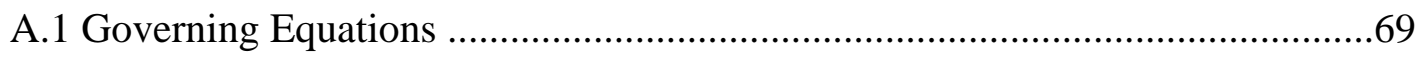

A.2 Finite Element Formulation ……………………………………………....

A.3 Solution Methodology ……………………………..................................

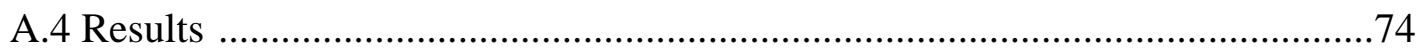

5.0.1 NACA 0012 Wing ..............................................................................74

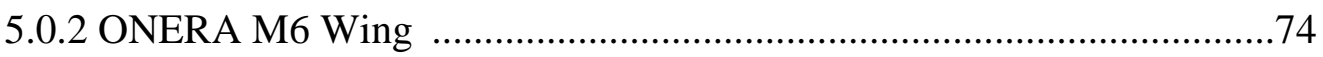

$\begin{array}{ll}\text { Vita } & 78\end{array}$ 


\section{List of Figures}

1. Pressure error for converging-diverging nozzle with purely subsonic flow.................19

2. Pressure error for converging-diverging nozzle with supersonic exit flow..................20

3. Pressure error for converging-diverging nozzle flow with a standing shock. ..............21

4. Element data distribution using triangular Bezier patches. ......................................40

5. Boundary element data distribution using Bezier segments....................................41

6. Computational regions in Ringleb flow domain used for analysis............................42

7. Generation of finite element meshes for Ringleb flow..........................................43

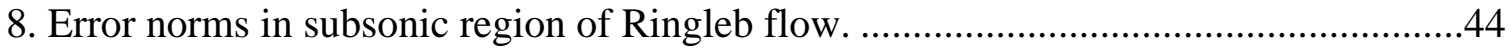

9. Error norms in supersonic region of Ringleb flow. ...............................................45

10. Geometry and grid for parabolic bump in a channel. .........................................46

11. Surface Mach number distribution for parabolic bump in a channel.........................47

12. Mach number contours for parabolic bump on a wall ..........................................48

13. Fine grid for inviscid flow over a NACA 0012 airfoil. ........................................49

14. Surface pressure distribution for subcritical NACA 0012 airfoil.............................50

15. Surface pressure distribution for transonic NACA 0012 airfoil...............................51

16. Solution domain for rotational Couette flow.......................................................52

17. Error in circumferential velocity for rotational Couette flow..................................53

18. Solution domain and baseline grid for flat plate flow..........................................54

19. Skin friction distributions for flat plate flow. ...................................................55

20. Sample grid for laminar flow over a NACA 0012 airfoil......................................56

21. Skin friction distributions for laminar flow over a NACA 0012 airfoil. ....................57 
22. Convergence histories for laminar flow over a NACA 0012 airfoil...........................58

23. Element data distribution using triangular Bezier patches. ....................................75

24. Mid-span surface pressure distribution for NACA 0012 wing.................................76

25. Surface pressure distribution for ONERA M6 wing at $65 \%$ span............................77 


\section{Chapter 1}

\section{Introduction}

High-Reynolds-number Navier-Stokes computations for complex aerodynamic configurations currently require vast amounts of computer resources for adequate resolution of the flow field. Indeed, the demands are so great that it is often not possible to perform complete and rigorous grid convergence studies for these configurations. State-of-the-art methods rely on linear data distributions in mesh cells resulting in at best second order accuracy. Methods based on higher order data distributions introduce additional computational complexity, but yield more accurate results, especially as the mesh is refined. Higher order methods have the potential to achieve solutions of much higher quality on coarser meshes compared to present state-of-the-art methods.

One of the most popular schemes for obtaining solutions on unstructured meshes is the finite volume scheme, in which the governing equations are solved in integral form over the discrete volumes formed by the cells of a mesh. Descriptions of various finite-volume schemes on unstructured meshes are given by Barth and Jesperson[1], Whitaker, et al.[2], Jameson, et al.[3, 4, 5], and Mavriplis and Jameson[6]. Barth[7] presents a detailed account of the implementation of finite volume schemes for the Euler and Navier-Stokes equations using efficient edge-based data structures. Finite volume schemes generally solve for quantities averaged over cells of the actual mesh in the case of cell-centered schemes or over cells of a dual mesh in the case of vertex schemes. In any event, in order to evaluate the residual, a polynomial data distribution must be reconstructed from these 
averaged quantities.

To achieve second order accuracy (as in the foregoing references), a linear distribution can be reconstructed in a cell using data from the cell's immediate neighbors. In contrast, to achieve higher than second order accuracy, a higher order distribution must be constructed in each cell, requiring information from more distant neighbors. This was done by Barth and Frederickson[8] for quadratic reconstruction (and hence third order accuracy). More recently, $\mathrm{Hu}$ and Shu[9] devised a fourth order scheme without expanding the third order stencil by requiring averaged quantities to match in all cells of the stencil. While these methods show promising results, extending them to even higher order accuracy will require further expansion of the stencil to still more distant neighboring cells. These stencils will be nonsymmetric in general and the reconstruction indices and coefficients must be stored for every cell in contrast to finite-element methods, in which interpolation coefficients are identical in every cell.

Halt[10] and Halt and Agarwal[11] used a variation of a finite volume scheme in which higher order polynomial data distributions were constructed locally in each cell using cell-averaged derivative information. To solve for the cell-averaged derivatives, the governing equations were extended to include either derivatives or moments of the governing equations. Halt demonstrated that significant gains in accuracy as well as efficiency could be achieved through the use of higher order methods. Halt also concluded that using moments of the governing equations was more robust than using the derivative method. Halt's moment method is similar to the Discontinuous Galerkin finite-element method described later in this chapter.

An alternative to the finite-volume formulation is the finite-element method. In this case, a polynomial data distribution is prescribed in each cell rather than reconstructing the distribution from averaged quantities. Finite element theory is described in detail by Zienkiewicz[12], Hughes[13], and Baker and Pepper[14]. In this method, the governing equations are solved in weak form by forming an inner product of the residual and a set of "trial" functions. As with finite difference and finite volume schemes, care must be taken to produce a stable scheme for the Euler and Navier-Stokes equations. 
Recently, a finite element method for solving hyperbolic systems, the "discontinuous Galerkin" (DG) method, has gained considerable popularity. In this method, the solution is allowed to have finite discontinuities at cell interfaces by prescribing independent sets of polynomial coefficients in each element. A Riemann solver is used to compute a unique flux at element interfaces and to provide an upwind formulation. Descriptions of the method are given by Cockburn, et al.[15, 16, 17] and Bey[18]. Atkins and Shu[19] applied the method to the linearized Euler equations, while Lowrie, et al.[20] and Bey and Oden[21] applied the method to the Euler equations and Bassi and Rebay[22, 23] applied the method to the Euler and Navier-Stokes equations.

A disadvantage of the DG method is that more unknowns are required to represent the double-valued solution on cell boundaries. For orders of accuracy less than or equal to 4 , the number of unknowns for the DG method is a factor of over 2 greater for triangles and nearly 5 greater for tetrahedra than a comparable continuous formulation.

By enforcing a continuous solution, stabilization of the system by means of a Riemann solver are precluded unless a discontinuous solution is somehow reconstructed. An alternative is to add either an explicit stabilizing dissipation to the residual itself or to modify the finite-element trial function. Brooks and Hughes[24] showed that these two approaches are equivalent in one dimension. Methods of this type fall into the general category of stabilized finite element methods. The theoretical basis for these methods will be outlined briefly below.

Given an operator $L$ and a forcing function $f$, the solution $u$ is sought which satisfies

$$
L u=f
$$

in a domain $\Omega$ subject to boundary conditions. Finite element methods solve equation 1 in the weak form given by

$$
\langle L u, v\rangle=\langle f, v\rangle
$$

where equation 2 is satisfied for all trial functions $v$. The inner product $\langle a, b\rangle$ is defined as

$$
\langle a, b\rangle=\int_{\Omega}(b \cdot a) d \Omega
$$

In a stabilized finite element method, the variational statement is modified such that 
consistency is preserved and stability is enhanced[25]. This preservation of consistency is a key feature of stabilized methods allowing high order accuracy. The modified weak statement is written as

$$
\langle L u, v\rangle+\sum_{K} \tau_{K}\left\langle L u-f, L^{\prime} v\right\rangle_{K}=\langle f, v\rangle
$$

where $\mathrm{K}$ indexes the elements, $\tau_{K}$ is a scaling parameter defined on each element, and $L^{\prime}$ is another differential operator which may or may not coincide with $L$. The only requirement on $\tau_{K}$ is that it vanishes as the grid is refined. Note that the inner product in the stabilizing term includes the entire residual.

In advective-diffusive systems, the Streamline Upwind/Petrov-Galerkin (SU/PG) scheme results when $L^{\prime}$ is equal to the advective part of the operator $L$. This method is presented in detail by Brooks and Hughes[24] and Hughes and Mallet[26, 27]. The method has been applied to the compressible Euler and Navier-Stokes equations by Soulaïmani and Fortin[29], Franca, et al.[30], and by Brueckner and Heinrich[31]. Carette, et al.[32] and Paillere, et al.[33] applied the method using multidimensional upwinding techniques and showed that in one dimension, the method derived in this way is identical to the original SU/PG method derived in [26]. While the SU/PG method has been applied extensively to linear data distributions in the literature, there seems to be no prior application of the method to higher order discretizations.

If $L^{\prime}=L$ in equation 4, the method is referred to as Galerkin Least-Squares (GLS). This method is described in detail by Hughes, et al.[28] for scalar advective-diffusive equations and by Shakib, et al.[34, 35] for the Euler and Navier-Stokes equations. Since the fundamental source of instability in the Euler and Navier-Stokes equations is the dominance of the advective terms, it is unclear that the additional complexity of the GLS method over the SU/PG method has any real benefit. Note, however, that for purely advective equations SU/PG and GLS are identical.

The SU/PG method would seem the most promising path to achieving a practical higher order scheme. In the following chapters, the SU/PG method will be described in 
detail and applied to higher order discretizations of inviscid flow in one dimension and inviscid and viscous flows in two dimensions. An appendix is included in which the method is applied to inviscid flows in three dimensions. The spatial convergence rate of the method will be established for the two-dimensional scheme by performing grid refinement studies and computing norms of the global solution error for Ringleb flow[36] and Couette flow[37]. 


\section{Chapter 2}

\section{The Streamline Upwind/Petrov-Galerkin Method}

\subsection{Scalar Advection - One Dimension}

Consider the steady-state scalar advection equation in one dimension:

$$
a \frac{d u}{d x}=0
$$

where $a$ is an advection speed and $u$ is the unknown solution. The Galerkin finite-element discretization of this equation is unstable, so an artificial dissipation term with coefficient $\bar{k}$ is added to stabilize the scheme, resulting in equation 6 below.

$$
a \frac{d u}{d x}=\frac{d}{d x} \bar{k} \frac{d u}{d x}
$$

Now consider a Galerkin formulation on the modified equation:

$$
\int_{0}^{L} w\left(a \frac{d u}{d x}-\frac{d}{d x} \bar{k} \frac{d u}{d x}\right) d x=0
$$

where $w$ is the Galerkin weight function. Integrating the artificial dissipation term by parts yields the following: 


$$
\int_{0}^{L} w a \frac{d u}{d x} d x-\left.w \bar{k} \frac{d u}{d x}\right|_{0} ^{L}+\int_{0}^{L} \frac{d w}{d x} \bar{k} \frac{d u}{d x} d x=0
$$

By stipulating that the dissipation coefficient $\bar{k}$ vanishes at the boundaries, and selecting the following form for $\bar{k}$

$$
\bar{k}=a \tau a
$$

where $\tau$ is a scaling parameter, the residual equation can be written in the following form:

$$
\int_{0}^{L}\left(w+\frac{d w}{d x} a \tau\right) a \frac{d u}{d x} d x=0
$$

The effect of the artificial dissipation is seen to be equivalent to the addition of a perturbation to the Galerkin weight function. The Euler-Lagrange equation corresponding to equation 10 is identical to equation 5; thus, the exact solution satisfies the residual equation and the method is consistent.

It has been shown in reference 24 that the following form for the parameter $\tau$ will yield a nodally exact solution to equation 5:

$$
\tau=\frac{\Delta x}{2|a|}
$$

where $\Delta x$ is the element size. For linear data on a uniform grid, the above definition of $\tau$ results in the following discrete equation for node $i$ :

$$
\frac{a+|a|}{2}\left(u_{i}-u_{i-1}\right)+\frac{a-|a|}{2}\left(u_{i+1}-u_{i}\right)=0
$$

which is identical to the discretization of Courant, Isaacson and Reeves[38].

The following sections summarize the extension of this formulation to multiple dimensions and to systems of equations. While the residual equation can be straightforwardly extended to these cases, the form of the parameter $\tau$ for multidimensional equations and systems is rather ad hoc and still the subject of research (see e.g. 29, 32, 39). 


\subsection{Advective Systems - One Dimension}

The extension of the SUPG scheme to one-dimensional systems is fairly straightforward. The governing equations can be written as

$$
\frac{d}{d x} F(Q)=0
$$

where $F$ is an advective flux vector which is, in general, a nonlinear function of the solution vector $Q$. The flux Jacobian $A$ is defined as a matrix whose elements are the derivatives of the components of the flux vector with respect to the components of the solution vector (i.e. $A=\partial F / \partial Q$ ). The governing equations can now be written in quasi-linear form as

$$
A \frac{d Q}{d x}=0
$$

The system can now be diagonalized by executing the matrix transformation

$$
A=T \Lambda T^{-1}, \Lambda=\operatorname{diag}\left(\lambda_{1}, \ldots, \lambda_{n}\right)
$$

where $\lambda_{i}$ are the eigenvalues of the Jacobian matrix $A$ and $T$ and $T^{-1}$ are formed by its right and left eigenvectors, respectively. The number of equations in the system is represented by $n$. The scalar SU/PG scheme is applied to each of the resulting $n$ scalar equations, resulting in $n$ parameters $\tau_{i}$ given by

$$
\tau_{i}=\frac{\Delta x}{2\left|\lambda_{i}\right|}
$$

By applying the inverse matrix transformation, the residual equation becomes

$$
\int_{0}^{L}\left(w+\frac{d w}{d x} A \tau\right) \frac{d F}{d x} d x=0
$$

where the parameter $\tau$ is now matrix-valued and is given by

$$
\tau=T^{-1} \operatorname{diag}\left(\tau_{1}, \ldots, \tau_{n}\right) T=\frac{\Delta x}{2}|A|^{-1} .
$$




\subsection{Scalar Advection - Multiple Dimensions}

In multiple dimensions, the advection operator is formed as a dot product between an advection vector and the solution gradient as in equation 19.

$$
a_{i} \frac{\partial u}{\partial x_{i}}=0
$$

where repeated indices indicate summation over the spatial dimensions. The resulting weak statement is:

$$
\int_{\Omega}\left[w+\left(\frac{\partial w}{\partial x_{j}} a_{j}\right) \tau\right]\left(a_{i} \frac{\partial u}{\partial x_{i}}\right) d \Omega=0 .
$$

As noted in reference 27, the direction of upwinding need not coincide with the streamline and can, in fact, contain any component perpendicular to the gradient of the solution. This leads to so-called "discontinuity capturing" operators[27]. Mizukami and Hughes[40] used this fact to construct a scheme to solve the scalar advection-diffusion problem with a linear interpolant on triangular elements that satisfies a maximum principle; however, this scheme has no extension to higher order interpolants, higher dimensions or different element shapes. In fact, no optimal upwinding direction has been formulated for these cases; thus, for simplicity, the upwind direction used in the current study coincides with the advection vector. This leads to the following definition for the (scalar) parameter $\tau$ :

$$
\tau=\left[\sum_{i}\left(\frac{\partial \xi_{i}}{\partial x_{j}} a_{j}\right)^{2}\right]^{-\frac{1}{2}}
$$

where the metric terms $\partial \xi_{i} / \partial x_{j}$ result from the element coordinate transformation and form the multidimensional analog of the one-dimensional length scale $\Delta x$. 


\subsection{Advective Systems - Multiple Dimensions}

A multidimensional advective system of equations can be written in the following form:

$$
\frac{\partial}{\partial x_{i}} F_{i}(Q)=0
$$

where $F_{i}$ is the $n$-component flux vector in the $i$ direction and repeated indices denote summation. As in the one-dimensional case, the system can be expressed in quasi-linear form as follows:

$$
A_{i} \frac{\partial Q}{\partial x_{i}}=0
$$

where $A_{i}$ is the flux Jacobian matrix in the $i$ direction. The SUPG weak statement is given by

$$
\int_{\Omega}\left[w+\left(\frac{\partial w}{\partial x_{j}} A_{j}\right) \tau\right]\left(A_{i} \frac{\partial Q}{\partial x_{i}}\right) d \Omega=0 .
$$

If the flux Jacobians $A_{i}$ are simultaneously diagonalizable, the system can be written as a set of $n$ scalar advection equations and the method of the previous section can be employed. Unfortunately, this is not the case in general and either an approximate diagonalization must be used or a matrix-valued analog of equation 21 must be devised. Examples of the former can be found in [32], in which wave models and characteristic decompositions are employed to allow the scalar SU/PG scheme to be used. The present study follows the latter strategy as in [26], wherein the (matrix-valued) parameter $\tau$ is defined by:

$$
\tau=\left(\sum_{i}\left|\frac{\partial \xi_{i}}{\partial x_{j}} A_{j}\right|\right)^{-1}
$$

where the metric terms $\partial \xi_{i} / \partial x_{j}$ are defined as in the previous section. 


\section{Chapter 3}

\section{Quasi-One-Dimensional Euler Equations}

\subsection{Governing Equations}

The equations solved are the one-dimensional Euler equations in conservation form with a source term to account for the variation of cross-sectional area. The equations can be written as

$$
\frac{\partial}{\partial x} F(Q)=S(Q)
$$

where

$$
Q=\left[\begin{array}{c}
\rho \\
\rho u \\
\rho e_{0}
\end{array}\right], F=\left[\begin{array}{c}
\rho u \\
\rho u^{2}+p \\
\rho u h_{0}
\end{array}\right], S=-\frac{1}{A} \frac{d A}{d x}\left[\begin{array}{c}
\rho u \\
\rho u^{2} \\
\rho u h_{0}
\end{array}\right]
$$

where $\rho$ is the density, $u$ is the speed, $p$ is the pressure, $e$ is the internal energy per unit mass, $h$ is the enthalpy per unit mass, $A$ is the cross-sectional area, and a subscript " 0 " indicates a stagnation condition.

The pressure is related to density and energy via the ideal-gas equation of state given by

$$
p=(\gamma-1) \rho e
$$

where $\gamma$ is the ratio of specific heats.

The equations are solved in nondimensional form by defining the following nondi- 
mensional quantities:

$$
\begin{aligned}
x^{*}=\frac{x}{L}, \rho^{*}=\frac{\gamma \rho}{\rho_{01}}, u^{*}=\frac{u}{c_{01}}, p^{*} & =\frac{p}{p_{01}}, T^{*}=\frac{T}{T_{01}}, e^{*}=\frac{e}{c_{01}^{2}}, h^{*}=\frac{h}{c_{01}^{2}}, \\
A^{*} & =\frac{A}{A_{t h}}
\end{aligned}
$$

where $c$ is the speed of sound, $A_{t h}$ is the minimum cross sectional area and the subscript " 01 " indicates a stagnation condition upstream of the inflow boundary. The speed of sound $c$ under the ideal-gas assumption is defined by

$$
c^{2}=\frac{\gamma p}{\rho}
$$

By substituting these expressions into the expressions for the inviscid and viscous fluxes, the nondimensional inviscid flux vector and the source term vector can be written as

$$
F^{*}=\left[\begin{array}{c}
\rho^{*} u^{*} \\
\rho^{*} u^{*}+p^{*} \\
\rho^{*} u^{*} h_{0}
\end{array}\right], S=-\frac{1}{A^{*}} \frac{d}{d x^{*}} A^{*}\left[\begin{array}{c}
\rho^{*} u^{*} \\
\rho^{*} u^{*} \\
\rho^{*} u^{*} h_{0}
\end{array}\right] .
$$

The nondimensional form of the ideal-gas equation of state is

$$
p^{*}=(\gamma-1) \rho^{*} e^{*}
$$

while the definition of the nondimensional speed of sound is

$$
c^{*^{2}}=\frac{\gamma p^{*}}{\rho^{*}}
$$

In the remaining sections of this chapter, the superscript is omitted from the nondimensional quantities for the sake of clarity.

\subsection{Finite Element Formulation}

Following the methodology of the previous section, the weak statement corresponding to the governing equations above is 


$$
\int_{x_{0}}^{x_{L}}\left(w+\frac{d w}{d x} A \tau\right)\left(\frac{d F}{d x}-S\right) d x=0 .
$$

After integration of the Galerkin part of the flux term by parts, this statement becomes

$$
\left.w F\right|_{x_{0}} ^{x_{L}}-\int_{x_{0}}^{x_{L}} \frac{d w}{d x} F d x-\int_{x_{0}}^{x_{L}} w S d x+\int_{x_{0}}^{x_{L}} \frac{d w}{d x} A \tau\left(\frac{d F}{d x}-S\right) d x=0
$$

At the upstream boundary, total pressure and total temperature are specified while density is evaluated just inside the boundary. At the downstream boundary, density and velocity are evaluated on the interior while a back pressure is prescribed. These boundary conditions are enforced weakly via the boundary flux term of equation 35.

\subsection{Solution Methodology}

The physical domain $x \in\left[x_{0}, x_{L}\right]$ is divided uniformly into elements of length $\Delta x$. This results in a constant transformation to a local element coordinate system in which the differential $d x$ and the derivative $d / d x$ are given by:

$$
d x=\Delta x d \xi, \frac{d}{d x}=\frac{1}{\Delta x} \frac{d}{d \xi}
$$

where $\xi$ is the barycentric coordinate in an element. The flow variables $Q$ are approximated by a Bezier curve over each element given by:

$$
Q(\xi)=\sum_{i=0}^{n} Q_{i} B_{i}^{n}(\xi)
$$

where the $Q_{i}$ are the discrete control points and the $B_{i}^{n}$ are the univariate Bernstein polynomials of degree $n$ given by

$$
B_{i}^{n}(\xi)=\frac{n !}{i !(n-i) !} \xi^{i}(1-\xi)^{n-i} .
$$

After writing the global integrals in equation 35 as a sum of element integrals and 
substituting the above data representation and coordinate transformation, the weak statement now becomes

$$
\left.B_{j} F\right|_{0} ^{L}+\sum_{\Omega_{e}}\left[-\int_{0}^{1} \frac{d B_{j}}{d \xi} F d \xi-\int_{0}^{1} B_{j} S \Delta x d \xi+\int_{0}^{1} \frac{d B_{j}}{d x} \operatorname{sgn} A\left(\frac{1}{\Delta x} \frac{d F}{d \xi}-S\right) d \xi\right]=0
$$

The element integrals appearing in equation 39 are evaluated numerically via Gaussian quadrature given by

$$
\int_{0}^{1} f(\xi) d \xi \approx \sum_{i=1}^{N} w_{i} f\left(\xi_{i}\right)
$$

where the weights $w_{i}$ and ordinates $\xi_{i}$ are given in [12].

To obtain the solution $Q$, Newton's method is applied to equation 39. First, the weak statement is linearized about an initial solution $Q^{n}$ to obtain a linear system of equations in the following form:

$$
\frac{\partial R_{j}}{\partial Q_{i}} \Delta Q_{i}+R_{j}=0
$$

where $R_{j}$ is the $j$-th weak statement evaluated on the initial solution $Q^{n}$ and $\Delta Q_{i}$ is the update to the control point $Q_{i}^{n}$.

The term $\partial R_{j} / \partial Q_{i}$ is the Jacobian matrix of the system and is evaluated approximately by the following equation:

$$
\begin{aligned}
& \left.\frac{\partial R_{j}}{\partial Q_{i}} \approx w A B_{j}\right|_{0} ^{L}+\sum_{\Omega_{e}}\left[-\int_{0}^{1} \frac{d B_{j}}{d \xi} A B_{i} d \xi-\int_{0}^{1} B_{j} \frac{\partial S}{\partial Q} B_{i} \Delta x d \xi+\right. \\
& \left.\int_{0}^{1} \frac{d B_{j}}{d x} \operatorname{sgn} A\left(\frac{1}{\Delta x} A \frac{\partial B_{i}}{\partial \xi}-\frac{\partial S}{\partial Q} B_{i}\right) d \xi\right]
\end{aligned}
$$

Note that this Jacobian is approximate because the dependencies of the flux Jacobian $A$ and the Jacobian of the source term $(\partial S / \partial Q)$ on the solution variables $Q$ are not included. Hence the resulting iterate will not recover the quadratic convergence of Newton's 
method.

Because neighboring elements are coupled only through their shared interface, the linear system represented by equation 41 is a block banded system with a maximum bandwidth of $2 n-13 \times 3$ blocks. This system is solved directly using banded Gaussian elimination to compute the solution updates $\Delta Q_{i}$. This process is repeated until the nonlinear system represented by equation 39 is satisfied to a given tolerance.

\subsection{Results}

A converging-diverging nozzle with a quadratic area distribution and expansion ratio of 3 was run with back pressures representing subsonic exit flow, supersonic exit flow, and a case with a shock in the nozzle. The area distribution is given by

$$
\frac{A}{A_{t h}}=1+\left(\frac{A_{e}}{A_{t h}}-1\right)\left(\frac{x}{L}\right)^{2}, \frac{x}{L} \in\left[-\frac{1}{2}, 1\right]
$$

The solution can be obtained exactly using the well known isentropic flow and normal shock relations summarized in [42]. The procedure for computing the exact solution begins by determining which of three possible flow regimes the flow is in. Given the expansion ratio and assuming sonic flow at the throat, two possible exit Mach numbers are computed using the following relation:

$$
\frac{A^{*}}{A}=\left(\frac{\gamma+1}{2}\right)^{\frac{\gamma+1}{2(\gamma-1)}} M\left(1+\frac{\gamma-1}{2} M^{2}\right)^{-\frac{\gamma+1}{2(\gamma-1)}}=f_{A}(M)
$$

where $\mathrm{M}$ is the Mach number and a superscript "*” indicates a sonic condition. This relation is solved for the exit Mach number using Newton iteration to achieve the required precision. It is a simple matter to choose starting conditions that will yield either the subsonic or the supersonic solution.

Next, the back pressure ratios required to produce these exit Mach numbers are computed using the following relation: 


$$
\frac{p}{p_{0}}=\left(1+\frac{\gamma-1}{2} M^{2}\right)^{-\frac{\gamma}{\gamma-1}}=f_{p}(M)
$$

The pressure ratios corresponding to the two exit Mach numbers divide the possible flows into three regimes: for a back pressure greater than or equal to that of the subsonic solution, the flow will be entirely subsonic; for a back pressure less than or equal to the supersonic solution, the flow downstream of the throat will be entirely supersonic; for all other values of back pressure, the flow will be sonic at the throat and will have either a normal shock downstream of the throat or an overexpanded supersonic exit flow.

For the first two cases, the exact solution can be computed using the isentropic flow relations in [42]. At the desired location, the area ratio is computed using equation 43. For supersonic exit flow, the flow at the throat is sonic and the supersonic solution of equation 44 determines the Mach number as described above. For subsonic flow, the exit Mach number is computed by substituting the back pressure into equation 45 and solving for the Mach number $M_{e}$. The area ratio is then computed using

$$
\frac{A^{*}}{A}=\left(\frac{A^{*}}{A_{e}}\right)\left(\frac{A_{e}}{A_{t h}}\right)\left(\frac{A_{t h}}{A}\right)=f_{A}\left(M_{e}\right)\left(\frac{A_{e}}{A_{t h}}\right)\left(\frac{A_{t h}}{A}\right)
$$

where the first factor is the area ratio obtained by evaluating equation 44 at the exit Mach number, the second factor is the constant expansion ratio, and the third factor is the area at the desired location. After substituting equation 46 into equation 44 and solving for the (subsonic) Mach number, the pressure at the desired location is obtained from equation 45 .

The third case is solved by first assuming that a normal shock exists downstream of the throat. Because the ratio of total pressures downstream and upstream of the shock is equal to the ratio of the sonic-flow cross sectional areas upstream and downstream of the shock, it can be shown that

$$
f_{p}\left(M_{e}\right) f_{A}\left(M_{e}\right)=\left(\frac{p_{b}}{p_{01}}\right)\left(\frac{A_{e}}{A_{t h}}\right)
$$

This equation is solved via Newton iteration to obtain the exit Mach number $M_{e}$. The location of the shock is determined by calculating the ratio of the total pressure upstream 
and downstream of the shock:

$$
\frac{p_{02}}{p_{01}}=\left(\frac{p_{02}}{p_{b}}\right)\left(\frac{p_{b}}{p_{01}}\right)=f_{p}\left(M_{e}\right)\left(\frac{p_{b}}{p_{01}}\right)
$$

This ratio is also defined by the normal shock relation

$$
\frac{p_{02}}{p_{01}}=\left[\frac{\gamma+1}{2 \gamma M^{2}-(\gamma-1)}\right]^{\frac{1}{\gamma-1}}\left[\frac{(\gamma+1) M^{2}}{(\gamma-1) M^{2}+2}\right]^{\frac{\gamma}{\gamma-1}}=g_{p}(M)
$$

which is solved via Newton iteration for the upstream Mach number at the shock, $M_{s}$. Equation 44 now yields the area ratio at the shock. If this area ratio is less than the expansion ratio, equation 43 is used to compute the location of the shock, otherwise, the shock is downstream of the exit.

The solution upstream of the shock is now computed as previously described by solving equation 44 for the (supersonic) Mach number. Downstream of the shock, the area ratio must be adjusted to account for losses through the shock as follows:

$$
\frac{A^{*}}{A}=\left(\frac{A_{2}^{*}}{A_{1} *}\right)\left(\frac{A_{1}^{*}}{A}\right)=g_{p}\left(M_{s}\right)\left(\frac{A_{t h}}{A}\right)
$$

The subsonic solution of equation 44 gives the Mach number at the desired location. The calculation of the pressure must also account for losses across the shock as follows:

$$
\frac{p}{p_{01}}=\left(\frac{p_{02}}{p_{01}}\right)\left(\frac{p}{p_{02}}\right)=g_{p}\left(M_{s}\right) f_{p}(M)
$$

Three different values of back pressure were used representing the three flow regimes described previously. The first case, at a back pressure ratio of 0.98 , represents a purely subsonic unchoked flow. The second case, at a back pressure of 0.28 , represents purely supersonic flow downstream of the throat. The final case, at a back pressure of 0.88 , represents a flow with a shock between the throat and exit. Several different orders of accuracy were used and the integrated $L^{2}$ error in the pressure distribution defined by 


$$
L^{2}=\int_{x_{0}}^{x_{L}}[p(x)-\hat{p}(x)]^{2} d x
$$

where $\hat{p}$ is the exact solution, was calculated for several different grid sizes.

The first case is a purely subsonic flow resulting from a back pressure ratio of 0.98 . Figure 1 shows the integrated norm of the pressure error versus the number of degrees of freedom in the problem. Design accuracy was verified up to 9th order (beyond this point 64-bit floating point numbers lack sufficient precision to resolve the spatial convergence rate of the scheme). Note that up to about degree 5 (order of accuracy 6) there are significant gains to increasing the order of accuracy as the 6th order result can be up to 5 orders of magnitude more accurate than the 2 nd order result. Also note that for linear data the scheme is superconvergent and results in a 3rd order solution.

Figure 2 shows pressure error for a case with supersonic exit flow. Note that for linear data the scheme is no longer superconvergent, but otherwise the same trends in accuracy are observed up to fifth order.

For the case of a normal shock in the nozzle, all linear schemes are at best first order accurate globally. Figure 3 shows the distribution of pressure error for a 4 th order solution. The parameter $J$ is the number of elements. Note that while the upstream flow is achieving design accuracy, the solution is at best first order not only at the shock but also downstream of the shock. This problem was discovered by Casper and Carpenter[41] and is also observed in these results.

These results verify that the SU/PG scheme as formulated for linear solution data (i.e. 2nd order schemes) can be used without modification to achieve higher order accuracy for smooth flows. 


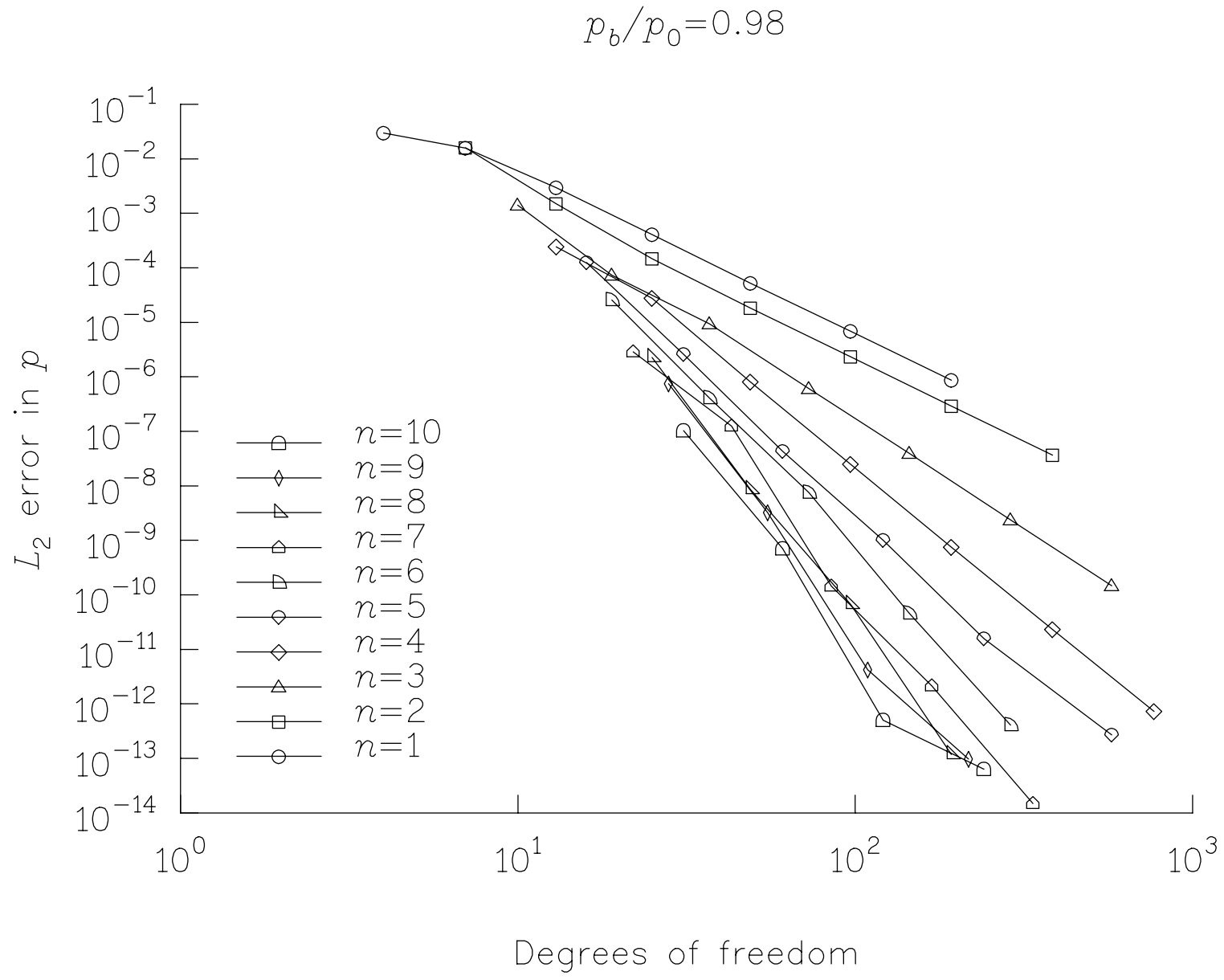

Figure 1. Pressure error for converging-diverging nozzle with purely subsonic flow. 


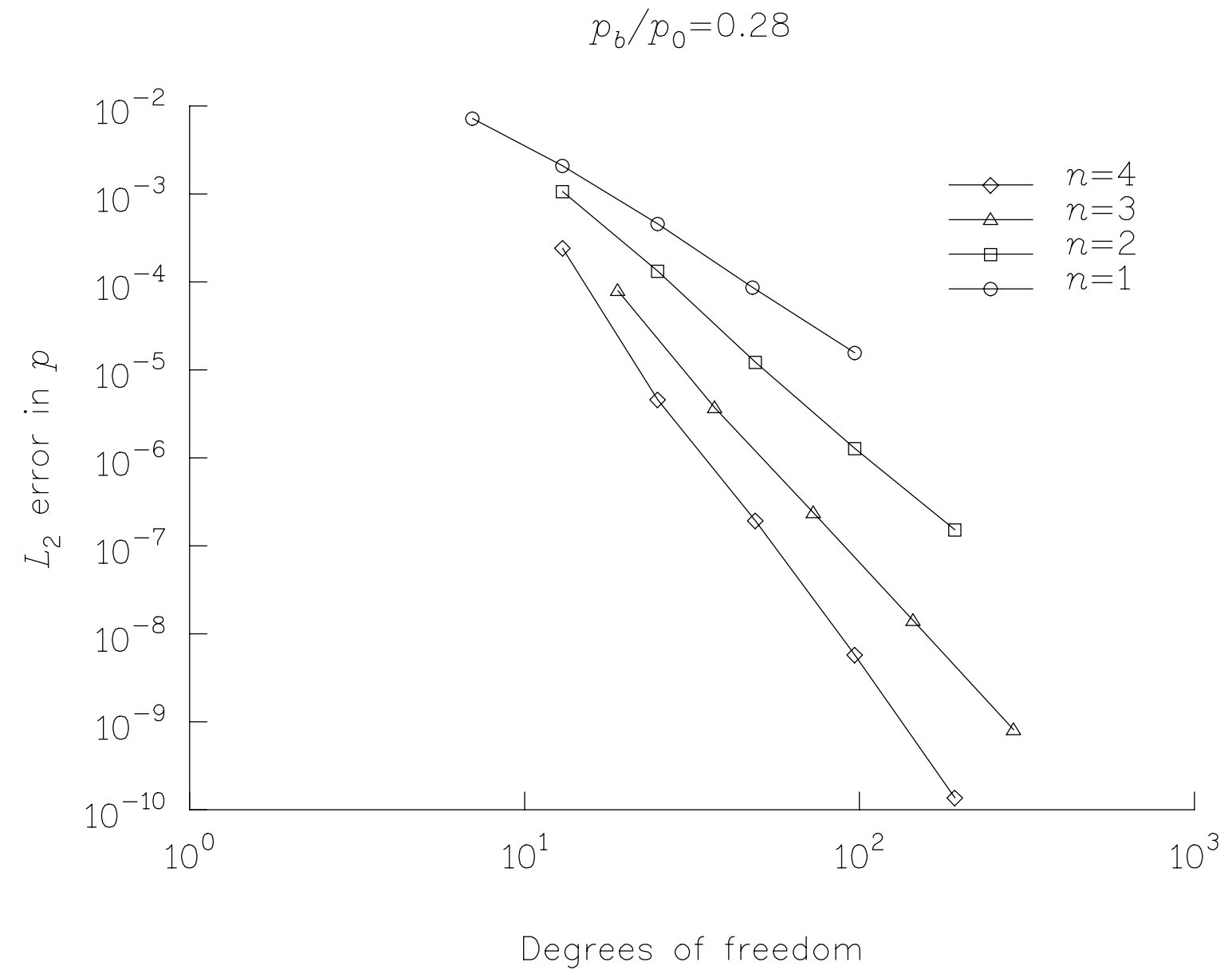

Figure 2. Pressure error for converging-diverging nozzle with supersonic exit flow. 


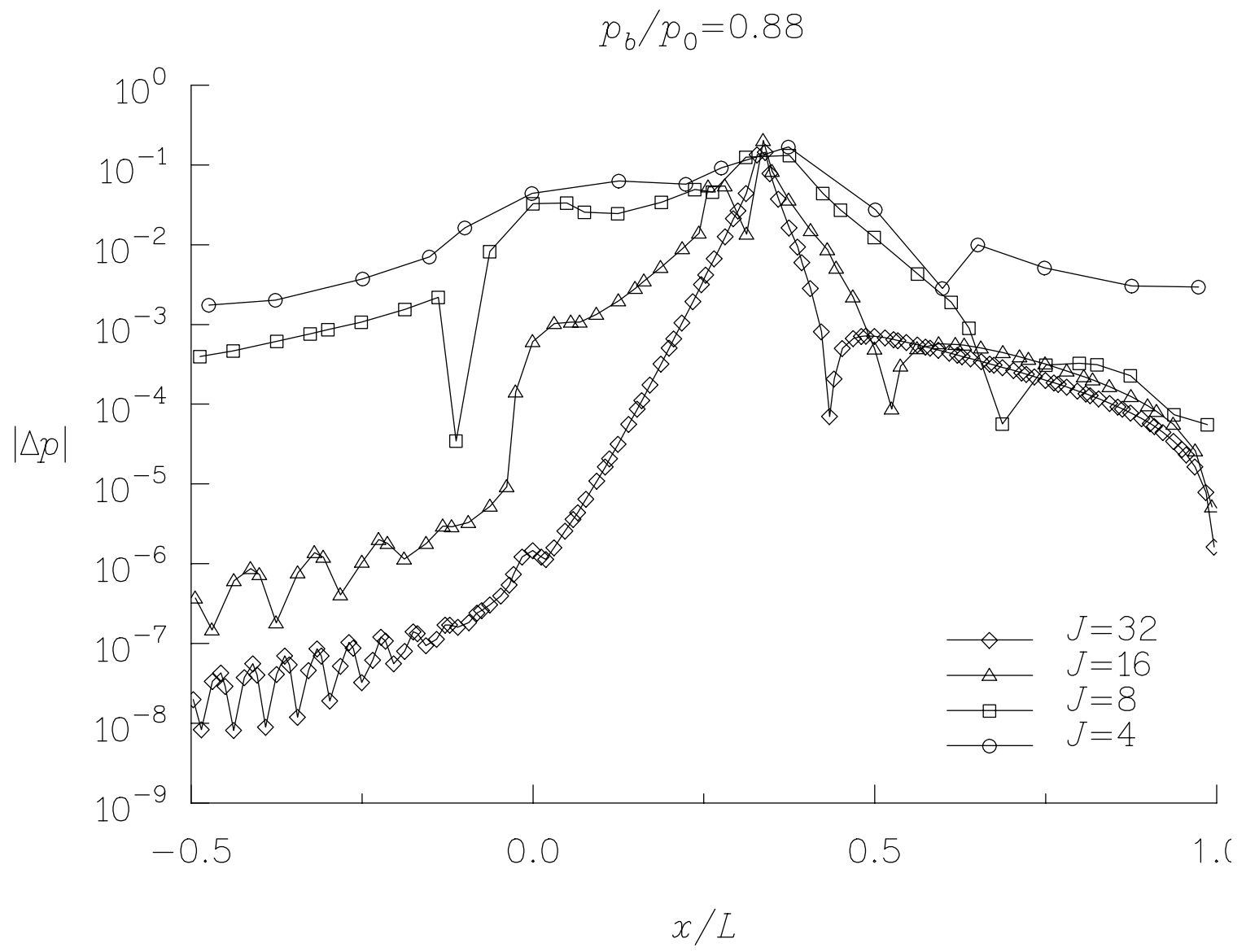

Figure 3. Pressure error for converging-diverging nozzle flow with a standing shock. 


\section{Chapter 4}

\section{Two-Dimensional Navier-Stokes Equa- tions}

\subsection{Governing Equations}

The governing equations are the steady-state Navier-Stokes equations in conservation form given by

$$
\frac{\partial F}{\partial x}+\frac{\partial G}{\partial y}=\frac{\partial F_{v}}{\partial x}+\frac{\partial G_{v}}{\partial y}
$$

where

$$
Q=\left[\begin{array}{c}
\rho \\
\rho u \\
\rho v \\
\rho e_{0}
\end{array}\right], F=\left[\begin{array}{c}
\rho u \\
\rho u^{2}+p \\
\rho u v \\
\rho u h_{0}
\end{array}\right], G=\left[\begin{array}{c}
\rho v \\
\rho u v \\
\rho v^{2}+p \\
\rho v h_{0}
\end{array}\right]
$$

where $\rho$ is the density, $u$ and $v$ are the Cartesian components of the velocity vector, $p$ is the pressure, $e$ is the internal energy per unit mass, $h$ is the enthalpy per unit mass, and a subscript " 0 " indicates a stagnation condition. The viscous fluxes $F_{v}$ and $G_{v}$ are given by 


$$
F_{v}=\left[\begin{array}{c}
0 \\
\tau_{x x} \\
\tau_{x y} \\
u \tau_{x x}+v \tau_{x y}-q_{x}
\end{array}\right], G_{v}=\left[\begin{array}{c}
0 \\
\tau_{x y} \\
\tau_{y y} \\
u \tau_{x y}+v \tau_{y y}-q_{y}
\end{array}\right]
$$

where the viscous stresses $\tau_{x x}, \tau_{x y}$, and $\tau_{y y}$ are evaluated for a Newtonian fluid under the bulk viscosity assumption.

$$
\tau_{x x}=\mu\left(\frac{4}{3} \frac{\partial u}{\partial x}-\frac{2}{3} \frac{\partial v}{\partial y}\right), \tau_{x y}=\mu\left(\frac{\partial u}{\partial y}+\frac{\partial v}{\partial x}\right), \tau_{y y}=\mu\left(\frac{4}{3} \frac{\partial v}{\partial y}-\frac{2}{3} \frac{\partial u}{\partial x}\right)
$$

where $\mu$ is the molecular viscosity. The heat flux components $q_{x}$ and $q_{y}$ are given by Fourier's law:

$$
q_{x}=-k \frac{\partial T}{\partial x}, q_{y}=-k \frac{\partial T}{\partial y}
$$

where $k$ is the Fourier heat transfer coefficient and $T$ is the static temperature.

The pressure is related to density and energy via the ideal-gas equation of state given by

$$
p=(\gamma-1) \rho e
$$

while the following power law valid for air at temperatures from $300^{\circ} \mathrm{R}$ to $900^{\circ} \mathrm{R}[42]$ is used to relate viscosity to temperature

$$
\frac{\mu}{\mu_{r}}=\left(\frac{T}{T_{r}}\right)^{0.76}
$$

where the subscript $r$ denotes a reference condition.

The equations are solved in nondimensional form by defining the following nondimensional quantities:

$$
\begin{gathered}
x^{*}=\frac{x}{L}, y^{*}=\frac{y}{L}, \rho^{*}=\frac{\rho}{\rho_{\infty}}, u^{*}=\frac{u}{c_{\infty}}, v^{*}=\frac{v}{c_{\infty}}, p^{*}=\frac{p}{\rho_{\infty} c_{\infty}^{2}}, T^{*}=\frac{R T}{c_{\infty}^{2}}, e^{*}=\frac{e}{c_{\infty}^{2}}, \\
h^{*}=\frac{h}{c_{\infty}^{2}}, \mu^{*}=\frac{\mu}{\mu_{\infty}}, k^{*}=\frac{k}{k_{\infty}}
\end{gathered}
$$


where $R$ is the ideal-gas constant, $L$ is a reference length and the subscript " $\infty$ " indicates a condition in the free stream. By substituting these expressions into the expressions for the inviscid and viscous fluxes, the nondimensional inviscid flux vectors can be written as

$$
F^{*}=\left[\begin{array}{c}
\rho^{*} u^{*} \\
\rho^{*} u^{*}+p^{*} \\
\rho^{*} u^{*} v^{*} \\
\rho^{*} u^{*} h_{0}^{*}
\end{array}\right], G^{*}=\left[\begin{array}{c}
\rho^{*} v^{*} \\
\rho^{*} u^{*} v^{*} \\
\rho^{*} v^{2}+p^{*} \\
\rho^{*} v^{*} h_{0}^{*}
\end{array}\right]
$$

while the viscous fluxes can be written as

$$
F_{v}{ }^{*}=\frac{M_{\infty}}{R e}\left[\begin{array}{c}
0 \\
\tau_{x x}{ }^{*} \\
\tau_{x y}{ }^{*} \\
u^{*} \tau_{x x}{ }^{*}+v^{*} \tau_{x y}{ }^{*}-q_{x}{ }^{*}
\end{array}\right], G_{v}{ }^{*}=\frac{M_{\infty}}{R e}\left[\begin{array}{c}
0 \\
\tau_{x y}{ }^{*} \\
\tau_{y y}{ }^{*} \\
u^{*} \tau_{x y}{ }^{*}+v^{*} \tau_{y y}{ }^{*}-q_{y}{ }^{*}
\end{array}\right]
$$

where $R e$ is the Reynolds number defined by $R e=\left(\rho_{\infty}\left\|\bar{u}_{\infty}\right\| L\right) / \mu_{\infty}$ where $\left\|\bar{u}_{\infty}\right\|$ is the magnitude of the free-stream velocity vector and the nondimensional viscous stresses are given by

$$
\begin{gathered}
\tau_{x x}^{*}=\mu^{*}\left(\frac{4}{3} \frac{\partial}{\partial x^{*}} u^{*}-\frac{2}{3} \frac{\partial}{\partial y^{*}} \nu^{*}\right), \tau_{x y}^{*}=\mu^{*}\left(\frac{\partial}{\partial y^{*}} u^{*}+\frac{\partial}{\partial x^{*}} v^{*}\right), \\
\tau_{y y}{ }^{*}=\mu^{*}\left(\frac{4}{3} \frac{\partial}{\partial y^{*}} \nu^{*}-\frac{2}{3} \frac{\partial}{\partial x^{*}} u^{*}\right)
\end{gathered}
$$

The nondimensional heat fluxes are given by

$$
q_{x}{ }^{*}=-\frac{\gamma}{\gamma-1} \frac{1}{P r} k^{*} \frac{\partial}{\partial x^{*}} T^{*}, q_{y}{ }^{*}=-\frac{\gamma}{\gamma-1} \frac{1}{P r} k^{*} \frac{\partial}{\partial y^{*}} T^{*}
$$

where the Prandtl number is defined by $\operatorname{Pr}=\mu c_{p} / k$, where $c_{p}$ is the specific heat at constant pressure. Under the assumption that the Prandtl number $P r$ is constant, the nondimensional Fourier heat transfer coefficient $k^{*}$ is equal to the nondimensional viscosity $\mu^{*}$.

In the remaining sections of this chapter, the superscript is omitted from the nondi- 
mensional quantities for the sake of clarity.

\subsection{Finite Element Formulation}

The Petrov-Galerkin weak statement of equation 53 is given by:

$$
\int_{\Omega}\left[w+\left(\frac{\partial w}{\partial x} A+\frac{\partial w}{\partial y} B\right) \tau\right]\left[\frac{\partial F}{\partial x}+\frac{\partial G}{\partial y}-\frac{M_{\infty}}{R e}\left(\frac{\partial F_{v}}{\partial x}+\frac{\partial G_{v}}{\partial y}\right)\right] d \Omega=0
$$

where the matrix $\tau$ is defined as in chapter 2. By integrating the Galerkin terms by parts, the above weak statement becomes:

$$
\begin{aligned}
& \int_{\Gamma} w\left(F \hat{n}_{x}+G \hat{n}_{y}\right) d \Gamma-\frac{M_{\infty}}{\operatorname{Re}} \int_{\Gamma} w\left(F_{v} \hat{n}_{x}+G_{v} \hat{n}_{y}\right) d \Gamma-\int_{\Omega}\left(\frac{\partial w}{\partial x} F+\frac{\partial w}{\partial y} G\right) d \Omega+ \\
& \frac{M_{\infty}}{\operatorname{Re}} \int_{\Omega}\left(\frac{\partial w}{\partial x} F_{v}+\frac{\partial w}{\partial y} G_{v}\right) d \Omega+\int_{\Omega}\left(\frac{\partial w}{\partial x} A+\frac{\partial w}{\partial y} B\right) \tau\left[\frac{\partial F}{\partial x}+\frac{\partial G}{\partial y}-\frac{M_{\infty}}{\operatorname{Re}}\left(\frac{\partial F_{v}}{\partial x}+\frac{\partial G_{v}}{\partial y}\right)\right] d \Omega=0
\end{aligned}
$$

where $\hat{n}_{x}$ and $\hat{n}_{y}$ are the Cartesian components of the boundary surface unit normal vector $\hat{n}$. The fluxes in the boundary integrals are evaluated based on the boundary conditions as described in the following paragraphs.

The inviscid flux on the boundary can be written as:

$$
F \hat{n}_{x}+G \hat{n}_{y}=\left[\begin{array}{c}
\rho u \hat{n}_{x}+\rho v \hat{n}_{y} \\
\left(\rho u^{2}+p\right) \hat{n}_{x}+\rho u v \hat{n}_{y} \\
\rho u v \hat{n}_{x}+\left(\rho v^{2}+p\right) \hat{n}_{y} \\
\rho u h \hat{n}_{x}+\rho v h \hat{n}_{y}
\end{array}\right]=\left[\begin{array}{c}
\rho \bar{u} \cdot \hat{n} \\
\rho u \bar{u} \cdot \hat{n}+p \hat{n}_{x} \\
\rho v \bar{u} \cdot \hat{n}+p \hat{n}_{y} \\
\rho h \bar{u} \cdot \hat{n}
\end{array}\right]
$$

On both inviscid and no-slip surfaces, the normal velocity $\bar{u} \cdot \hat{n}$ vanishes, resulting in the following boundary flux: 


$$
F \hat{n}_{x}+G \hat{n}_{y}=\left[\begin{array}{c}
0 \\
p \hat{n}_{x} \\
p \hat{n}_{y} \\
0
\end{array}\right]
$$

where the pressure $p$ is evaluated just inside the boundary. For subsonic flow across an inflow/outflow boundary, the inviscid flux is evaluated by computing the normal velocity and speed of sound from two locally one-dimensional Riemann invariants given by

$$
R^{ \pm}=\bar{u} \cdot \hat{n} \pm \frac{2 c}{\gamma-1}
$$

The quantity $R^{-}$is evaluated using free-stream conditions while $R^{+}$is evaluated based on values just inside the domain. The normal velocity and speed of sound on the boundary are then given by

$$
(\bar{u} \cdot \hat{n})_{b}=\frac{1}{2}\left(R^{+}+R^{-}\right), c_{b}=\frac{\gamma-1}{4}\left(R^{+}-R^{-}\right)
$$

The velocity components on the boundary are found by decomposing the normal velocity (given by equation 70) and the tangential velocity into components resulting in the following expressions:

$$
u_{b}=u_{r}+\hat{n}_{x}\left[(\bar{u} \cdot \hat{n})_{b}-(\bar{u} \cdot \hat{n})_{r}\right], v_{b}=v_{r}+\hat{n}_{y}\left[(\bar{u} \cdot \hat{n})_{b}-(\bar{u} \cdot \hat{n})_{r}\right]
$$

where the subscript $r$ denotes a reference condition in the free stream for flow into the domain and just inside the boundary for flow out of the domain. Similarly, entropy on the boundary is calculated from free-stream quantities for flow into the domain and from interior values for outflow. The density on the boundary is then calculated as

$$
\rho_{b}=\left(\frac{c_{b}^{2}}{\gamma S_{b}}\right)^{\frac{1}{\gamma-1}}
$$

For supersonic inflow and outflow, the boundary flux vector is calculated entirely from quantities in the free stream and just inside the boundary, respectively.

The viscous flux vector on the boundary can be written as 


$$
F_{v} \hat{n}_{x}+G_{v} \hat{n}_{y}=\left[\begin{array}{c}
0 \\
\tau_{x x} \hat{n}_{x}+\tau_{x y} \hat{n}_{y} \\
\tau_{x y} \hat{n}_{x}+\tau_{y y} \hat{n}_{y} \\
\left(u \tau_{x x}+v \tau_{x y}\right) \hat{n}_{x}+\left(u \tau_{x y}+v \tau_{y y}\right) \hat{n}_{y}+k \nabla T \cdot \hat{n}
\end{array}\right]
$$

On inviscid and inflow/outflow boundaries, the viscous flux is assumed to be zero. On noslip surfaces the condition $u=v=0$ is strongly enforced, replacing the momentum equations on those surfaces. As a result, the second and third elements of the boundary flux vector are irrelevant, and the fourth reduces to $k \nabla T \cdot \hat{n}$. An adiabatic wall is assumed, so this term also vanishes. Thus, the integral of the viscous boundary flux vanishes on all boundaries.

\subsection{Solution Methodology}

\subsubsection{Discretization}

The physical domain $\Omega$ is divided into a set of nonoverlapping triangular elements $\Omega_{e}$ such that the entire domain is represented. The solution data $Q$ are represented by triangular Bezier patches in each element defined by

$$
Q(\xi, \eta)=\sum_{i=0}^{n} \sum_{j=0}^{n-i} Q_{i j} B_{i j}^{n}(\xi, \eta)
$$

where $\xi$ and $\eta$ are the local barycentric coordinates of the element and the $B_{i j}^{n}$ are the bivariate Bernstein polynomials of degree $n$ given by

$$
B_{i j}^{n}(\xi, \eta)=\frac{n !}{i ! j !(n-i-j) !} \xi^{i} \eta^{j}(1-\xi-\eta)^{n-i-j} .
$$

To accommodate curved boundaries, the coordinates of each triangular element are also represented by triangular Bezier patches of degree $n$. This results in a nonlinear coordinate transformation from the physical space to the element parameter space. Derivatives in physical space $(x, y)$ are transformed to element parameter space $(\xi, \eta)$ by 


$$
\frac{\partial}{\partial x}=\frac{\partial \xi}{\partial x \partial \xi}+\frac{\partial \eta}{\partial x} \frac{\partial}{\partial \eta}, \frac{\partial}{\partial y}=\frac{\partial \xi}{\partial y \partial \xi}+\frac{\partial \eta}{\partial y} \frac{\partial}{\partial \eta} .
$$

The metric terms appearing in equation 76 can be expressed in terms of the derivatives of the element coordinates as

$$
\frac{\partial \xi}{\partial x}=\frac{1}{J} \frac{\partial y}{\partial \eta}, \frac{\partial \xi}{\partial y}=-\frac{1}{J} \frac{\partial x}{\partial \eta}, \frac{\partial \eta}{\partial x}=-\frac{1}{J} \frac{\partial y}{\partial \xi}, \frac{\partial \eta}{\partial y}=\frac{1}{J} \frac{\partial x}{\partial \xi}
$$

where $J$ is the Jacobian of the element coordinate transformation defined by

$$
J=\frac{\partial x \partial y}{\partial \xi \partial \eta}-\frac{\partial x}{\partial \eta} \frac{\partial y}{\partial \xi}
$$

Finally, the volume differential $d \Omega_{e}$ is scaled by the Jacobian $J$ as follows:

$$
d \Omega_{e}=J d \xi d \eta
$$

Continuity of the solution across element interfaces is enforced by sharing control points along the interfaces as illustrated in figure $4 \mathrm{a}$. The $(i, j)$ indexing in equation 74 is converted to a single index as shown in figure $4 \mathrm{~b}$ by the following function:

$$
i_{\text {node }}=j(2 n-j+3) / 2+i
$$

The boundary $\Gamma$ of the domain $\Omega$ is divided into a finite number of line elements $\Gamma_{e}$, each of which corresponds to the edge of a triangular element adjoining the boundary as shown in figure 5. The coordinates and data on each of these elements is represented by a Bezier curve of degree $n$ as described in the previous chapter. Integrals over the boundary $\Gamma$ can now be written as sums of integrals over individual boundary elements. These element integrals are transformed into integrals over the local element parameter space.

The polynomial expression of the boundary element coordinates gives rise to a continuously varying unit normal along curved elements. The components of the element normal vector are given by

$$
n_{x}=\frac{\partial y}{\partial \xi}, n_{y}=-\frac{\partial x}{\partial \xi}
$$

Boundary element integrals are transformed according to 


$$
\int_{\Gamma_{e}} f(x, y) d \Gamma_{e}=\int_{0}^{1} f[x(\xi), y(\xi)] s d \xi
$$

where

$$
s=\sqrt{\left(\frac{\partial x}{\partial \xi}\right)^{2}+\left(\frac{\partial y}{\partial \xi}\right)^{2}} .
$$

Upon substitution of the foregoing domain and boundary transformations, the weak statement (equation 66) can now be written as

$$
\begin{aligned}
& \sum_{\Gamma_{e}}\left[\int_{0}^{1} B_{i j}^{n}\left(F \hat{n}_{x}+G \hat{n}_{y}\right) s d \xi-\frac{M_{\infty}^{1}}{R e} \int_{0}^{1} B_{i j}^{n}\left(F_{v} \hat{n}_{x}+G_{v} \hat{n}_{y}\right) s d \xi\right]+ \\
& \sum_{\Omega_{e}}\left[-\int_{0}^{11} \int_{0}^{-\eta}\left(\frac{\partial B_{i j}^{n}}{\partial \xi}+\frac{\partial B_{i j}^{n}}{\partial \eta} \tilde{G}\right) J d \xi d \eta+\frac{M_{\infty}}{R e} \int_{0}^{11} \int_{0}^{1-\eta}\left(\frac{\partial B_{i j}^{n}}{\partial \xi} \tilde{F}_{v}+\frac{\partial B_{i j}^{n}}{\partial \eta} \tilde{G}_{v}\right) J d \xi d \eta+\right. \\
& 11-\eta \\
& \left.\int_{0}^{1} \int_{0}^{n}\left(\frac{\partial B_{i j}^{n}}{\partial \xi} \tilde{A}+\frac{\partial B_{i j}^{n}}{\partial \eta} \tilde{B}\right) \tau\left(\tilde{A} \frac{\partial Q}{\partial \xi}+\tilde{B} \frac{\partial Q}{\partial \eta}\right) J d \xi d \eta\right]=0
\end{aligned}
$$

where

$$
\tilde{F}=\frac{\partial \xi}{\partial x} F+\frac{\partial \xi}{\partial y} G, \tilde{G}=\frac{\partial \eta}{\partial x} F+\frac{\partial \eta}{\partial y} G
$$

are the transformed inviscid flux vectors and $\tilde{A}$ and $\tilde{B}$ are the corresponding flux Jacobians. The transformed viscous fluxes $\tilde{F}_{v}$ and $\tilde{G}_{v}$ are similarly defined. Note that the contribution of the viscous terms to the Petrov-Galerkin part of the weak statement has been neglected. These terms involve derivatives of the metric terms and are therefore difficult to compute. Reference 43 presents a local reconstruction technique to represent this contribution that may be incorporated in future work. As will be seen in the results, this omission has no impact on the order properties of the scheme.

The integrals appearing in equation 84 are evaluated numerically using the Gaussian quadrature rules of [44] for triangular elements and [12] for the line elements on the boundary. Finite element theory dictates that numerical quadrature must integrate polyno- 
mials of degree $2(n-m)$ exactly to preserve the convergence properties of the scheme[12]. Here $n$ is the degree of the interpolant and $m$ is the highest order derivative appearing in the integrand. This means that quadrature rules for triangular elements must be exact to degree $2(n-1)$ while boundary quadrature must be exact to degree $2 n$. Gaussian quadrature results in the minimum computational work for a given degree of accuracy, but availability of quadrature rules for triangular elements limits the scheme to fifth order accuracy.

\subsubsection{Solution Procedure}

The solution $Q$ is obtained using an approximate Newton method. First, the weak statement (equation 84) is approximately linearized about an initial solution $Q^{n}$ to give

$$
\left(\frac{M_{i j}}{\Delta t}+\frac{\partial R_{j}}{\partial Q_{i}}\right) \Delta Q_{i}+R_{j}=0
$$

where a pseudotime term has been added to improve diagonal dominance and allow more robust convergence. The mass matrix $M_{i j}$ is defined as

$$
M_{i j}=\sum_{\Omega_{e}} S_{e} \int_{0}^{11} \int_{0}^{-\eta} B_{k l}^{n} B_{q r}^{n} d \xi d \eta
$$

where $S_{e}$ is the element area. Note that this is an approximation of the true mass matrix, but time accuracy is not at issue, and the approximation allows the integral to be evaluated analytically and independent of the element shape - thus it can be precomputed and stored.

The system of linear equations represented by equation 86 is solved using the Generalized Minimum Residual method (GMRES) described in [45], which computes the solution of a general linear system iteratively by projecting the residual onto vectors in the Krylov subspace (An overview of Krylov subspace methods is given by Saad[46]). The GMRES algorithm yields an exact solution if all the Krylov vectors are used; however, in practice a subset of these vectors must be chosen to minimize storage requirements. Most 
implementations of the algorithm allow the solution to be restarted when the allotted storage for the Krylov vectors is exhausted. The implementation of the algorithm used in this work allows specification of the number of Krylov vectors to store, the number of restarts permitted, and a tolerance on the residual to use as a stopping criterion. Unless otherwise noted, all the test cases presented in this chapter stored 20 Krylov vectors, allowed one restart, and solved the system to a tolerance of 0.01 .

The GMRES algorithm does not require explicit knowledge of the matrix of the linear system - only the product of the matrix with the vector $\Delta Q_{i}$ is required. This allows the product of the Jacobian $\partial R_{j} / \partial Q_{i}$ and the solution update $\Delta Q_{i}$ to be written as a finitedifference expression as described in reference 47 and given by

$$
\frac{\partial R_{j}}{\partial Q_{i}} \Delta Q_{i} \approx \frac{R_{j}(Q+\varepsilon \Delta Q)-R_{j}(Q)}{\varepsilon}
$$

where $\varepsilon$ is a constant chosen such that the norm of $\varepsilon \Delta Q$ is the square root of machine precision.

The performance of the GMRES algorithm depends, in general, on the use of a suitable preconditioner. The preconditioner should approximate the inverse of the matrix, but must be simpler to solve. The simplest preconditioning is diagonal or Jacobi preconditioning, in which only diagonal terms of the matrix are retained and the resulting diagonal system is solved. Other forms of preconditioning such as incomplete LU factorization[48] or least-squares approximate inverse techniques[49, 50, 51] can improve convergence of the GMRES algorithm at the expense of increased computational complexity and storage[48].

The preconditioning used in this work is a block-diagonal preconditioning in which $4 \times 4$ blocks are retained on the diagonal of the matrix. This preconditioning is easily solved by inverting a 4x4 matrix for each degree of freedom. The block-diagonal matrix is represented by

$$
\frac{M_{k k}}{\Delta t}+\frac{\partial R_{k}}{\partial Q_{k}}
$$

where the diagonal block of the system Jacobian matrix $\partial R_{k} / \partial Q_{k}$ is approximated by 


$$
\begin{aligned}
\frac{\partial R_{k}}{\partial Q_{k}} \approx & \sum_{\Gamma_{e}}\left[\int_{0}^{1} B_{i j}^{n}\left(A \hat{n}_{x}+B \hat{n}_{y}\right) B_{i j}^{n} s d \xi-\frac{M_{\infty}}{R e} \int_{0}^{1} B_{i j}^{n}\left(A_{v} \hat{n}_{x}+B_{v} \hat{n}_{y}\right) B_{i j}^{n} s d \xi\right]+ \\
& \sum_{\Omega_{e}}\left[-\int_{0}^{11-\eta} \int_{0}^{n}\left(\frac{\partial B_{i j}^{n}}{\partial \xi}+\frac{\partial B_{i j}^{n}}{\partial \eta}\right) B_{i j}^{n} J d \xi d \eta+\frac{M_{\infty}}{R e} \int_{0}^{11-\eta} \int_{0}^{1-} \frac{\partial B_{i j}^{n}}{\partial \xi} \tilde{A}_{v}+\frac{\partial B_{i j}^{n}}{\partial \eta} \tilde{B}_{v}\right) B_{i j}^{n} J d \xi d \eta+ \\
& 11-\eta \\
& \left.\int_{0}^{11} \int_{0}^{n}\left(\frac{\partial B_{i j}^{n}}{\partial \xi} \tilde{A}+\frac{\partial B_{i j}^{n}}{\partial \eta} \tilde{B}\right) \tau\left(\tilde{A} \frac{\partial B_{i j}^{n}}{\partial \xi}+\tilde{B} \frac{\partial B_{i j}^{n}}{\partial \eta}\right) J d \xi d \eta\right]
\end{aligned}
$$

Note that the dependence of the flux Jacobians and of $\tau$ on the solution $Q$ is neglected in this approximation.

\subsection{Inviscid Flow Results}

\subsubsection{Ringleb Flow}

The first case presented is that of Ringleb's flow, which is presented in detail by Chiocchia[36]. This flow is an exact solution of the Euler equations for an ideal gas obtained by using a hodograph transformation. The equations are transformed from the Cartesian $(x, y)$ coordinate system to the $(q, \theta)$ hodograph plane where $q$ is the velocity magnitude and $\theta$ is the angle the velocity vector makes with a reference axis. The momentum equations can be expressed in stream function form as

$$
q^{2} \frac{\partial^{2} \psi}{\partial q^{2}}+q\left(1+\frac{q^{2}}{c^{2}}\right) \frac{\partial \psi}{\partial q}+\left(1-\frac{q^{2}}{c^{2}}\right) \frac{\partial^{2} \psi}{\partial \theta^{2}}=0
$$

where $\psi$ is the stream function defined such that the Cartesian velocity components are given by

$$
u=\frac{\rho_{r}}{\rho} \frac{\partial \psi}{\partial y}, v=-\frac{\rho_{r}}{\rho} \frac{\partial \psi}{\partial x}
$$

where the subscript $r$ indicates an arbitrary reference condition. This choice of stream function identically satisfies the continuity equation. 
The particular solution representing Ringleb flow is given by

$$
\psi=\frac{1}{\bar{q}} \sin \theta
$$

where the overbar indicates division by a reference quantity. The streamlines for this solution are given by

$$
x=\frac{1}{2 \bar{\rho}}\left(\frac{1}{\bar{q}^{2}}-\frac{2}{k^{2}}\right)+\frac{J}{2}, y= \pm \frac{1}{k \bar{\rho} \bar{q}} \sqrt{1-\left(\frac{\bar{q}}{k}\right)^{2}}
$$

where

$$
\begin{aligned}
k & =1 / \psi \\
J & =\frac{1}{\bar{c}}+\frac{1}{3 \bar{c}^{3}}+\frac{1}{5 \bar{c}^{5}}-\frac{1}{2} \log \frac{1+\bar{c}}{1-\bar{c}} \\
\bar{c} & =\sqrt{1-\frac{\gamma-1}{2} \bar{q}^{2}} \\
\bar{\rho} & =\bar{c}^{2 /(\gamma-1)}
\end{aligned}
$$

The geometry is determined a posteriori by choosing two streamlines to serve as solid walls along with lines of constant velocity as inflow and outflow boundaries. A typical geometry for this flow is shown in figure 6, where the solid walls are formed by streamlines corresponding to $k=0.8$ and $k=1.6$, and the outflow boundary is given by $\bar{q}=0.4$.

In order to avoid the necessity of generating a high-order discretization of the curved boundaries, triangular regions were selected from the traditional Ringleb flow domain. One region lies entirely within the subsonic portion of the flow, while the other region is within the supersonic region as illustrated in figure 6. Finite element meshes are generated in each region by uniformly subdividing the region as shown in figure 7a. Additional degrees of freedom required for the higher order interpolants are added via linear interpolation of the mesh coordinates as illustrated in figure $7 \mathrm{~b}$. The exact solution was supplied as a strongly enforced Dirichlet boundary condition.

Figure 8 shows the integrated norm of the error in the solution variables as a function of the number of degrees of freedom. Note that design accuracy has been obtained up to 
5th order. Similar behavior is noted in figure 9, which shows the integrated error norm for the supersonic region.

\subsubsection{Bump on a Wall}

A simple case incorporating curved boundaries is shown in figure 10a. Four quadratic segments form a bump on a wall whose height is $10 \%$ of its length. The segments have continuous derivatives at their junction points. The mesh depicted in figure 10a is a baseline mesh which was uniformly subdivided to control the number of unknowns in the problem in a fashion similar to that used for Ringleb flow described in the previous section. After subdividing and distributing additional degrees of freedom, the control points on the lower wall were moved to match the Bezier representation of the geometry. An example is given in figure $10 \mathrm{~b}$ for a subdivision factor of 2 and cubic data.

Figure 11a shows the Mach number distribution along the lower wall for a freestream Mach number of 0.4. Note the considerable difference in the 2nd and 3rd order solutions both at the peak and downstream to the outflow boundary. The SU/PG solutions are also compared with results obtained from an implementation of a second order finite volume scheme known as FUN2D[52]. Note that the finite volume results agree with the second order SU/PG scheme at the peak, but the finite volume results are much more accurate in the area of decelerating flow on the aft side of the bump. The second order SU/PG scheme generates a significantly larger amount of entropy near the body than the finite volume scheme as shown by the flow-field Mach number contours in figure 12 . Note that the contours for the finite volume solution smoothly approach the body while those for the SU/PG solution show a significant jump in Mach number near the wall. This is an indication that while the SU/PG scheme achieves higher order accuracy, the relative error levels may be improved by deriving an improved SU/PG formulation.

\subsubsection{NACA 0012 Airfoil}

Figure 13 shows a section of a mesh around a NACA 0012 airfoil obtained using the 
grid generation method of Marcum, et al.[53, 54]. The finest grid, depicted in the figure, had 96 points distributed on the airfoil surface and 32 points distributed along a circular outer boundary with a radius of 20 chord lengths. Two coarser meshes were generated by selecting alternating points on the boundaries and retriangulating the volume.

The NACA 4-digit thickness profile[55] is given by

$$
y= \pm \frac{t}{0.2}\left(0.29690 \sqrt{x}-0.12600 x-0.35160 x^{2}+0.28430 x^{3}-0.10150 x^{4}\right)
$$

where $t$ is the maximum thickness. By parametrizing $x$ as $x=\xi^{2}, y$ can be written in terms of $\xi$ as

$$
y= \pm \frac{t}{0.2}\left(0.29690 \xi-0.12600 \xi^{2}-0.35160 \xi^{4}+0.28430 \xi^{6}-0.10150 \xi^{8}\right)
$$

Thus the thickness distribution can be exactly represented by an 8th-order parametric Bezier curve. To generate higher order finite element meshes, this defining curve was subdivided to match the domain of each edge on the surface of an existing mesh and a leastsquares procedure was used to obtain the control points for the desired accuracy. The endpoints of each edge were forced to match the surface exactly.

The first case was run at a Mach number of 0.63 and an angle of attack of 2 degrees. At these conditions, the flow is completely subsonic. Figure 14a shows the surface pressure distribution obtained using the SU/PG scheme for several orders of accuracy. Each case has approximately the same number of degrees of freedom. Note the slight difference in pressure between the 2nd and 3rd order solutions. A comparison of the SU/PG results with results obtained from FUN2D are shown in figure 14b. The second order SU/PG results are in close agreement with the finite volume results.

A second case at a Mach number of 0.8 and an angle of attack of 1.25 degrees was run and surface pressure distributions obtained using FUN2D and the second and third order SU/PG schemes is shown in figure 15. At these conditions, the flow is transonic and shocks exist on both the upper and lower surfaces of the airfoil. Note that the two second order schemes are in agreement and that the third order SU/PG scheme captures the upper surface shock more sharply. 


\subsection{Laminar Viscous Flow Results}

\subsubsection{Couette Flow}

To verify the formal accuracy of the scheme, a rotational Couette flow was computed. The solution domain is depicted in figure 16a. Two concentric cylinders are in relative angular motion inducing fluid motion in the annular region. An analytic solution for the angular velocity exists for incompressible flow and is given by:

$$
u_{\theta}=A r+\frac{B}{r}
$$

where $A$ and $B$ are constants depending on the geometry and on the boundary conditions and $r$ is the distance from the common center of the cylinders. This solution also applies to compressible flows as long as viscosity is constant. In a real flow, the temperature dependence of viscosity couples the momentum and energy equations, but for the purpose of establishing the accuracy of the scheme, this approximation will suffice.

Grids were generated by distributing Lagrange points for triangular elements along lines of constant $r$ and $\theta$ and then converting to the required Bezier description. A typical grid for a second-order calculation is shown in figure 16b. Figure 17 shows the integrated $L_{2}$ error in the circumferential velocity for a Couette flow where $r_{1}=1, r_{2}=4$, $\omega_{1}=0.2$ and $\omega_{2}=0$. This particular choice of parameters results in an exact solution for the circumferential velocity of

$$
u_{\theta}=\frac{1}{75}\left(-r+\frac{16}{r}\right)
$$

where the $1 / r$ term dominates. The Mach number at the inner cylinder $(0.2)$ and the Reynolds number (500) were chosen to be relatively low to avoid violating the assumptions of constant viscosity and laminar flow. The parameter $n$ indicates the degree of the basis functions. Design accuracy is confirmed up to fifth order. The case of quadratic data appears to show superconvergence, but this result may be peculiar to this case (it is sus- 
pected that the error may simply have no third-order components).

\subsubsection{Flat Plate}

The first case of practical interest is that of flow past a flat plate. Figure 18 shows the solution domain and an initial discretization that forms the basis of all the following calculations. For each case, the finite-element grid is characterized by two parameters $N$ and $n$. The refinement parameter $N$ indicates how many subdivisions of the baseline grid were performed, while the parameter $n$ is the degree of the interpolating polynomial. The number of degrees of freedom in the calculation is linearly related to the product of these two parameters.

Since the Riemann-invariant boundary condition is strictly applicable only to inviscid flows, the abutment of a viscous surface and an outflow boundary results in significant error over much of the plate; therefore a different boundary formulation is used. At the inflow, total pressure, total temperature and normal velocity are specified while static pressure is evaluated just inside the boundary. At the outflow, a back pressure is specified while the energy and velocity are evaluated just inside the boundary.

Figure 19a shows skin friction distributions for several cases at a Mach number of 0.3 and a Reynolds number of 500. The well known incompressible solution of Blasius[56] is shown for comparison. The flow conditions were chosen so as to compare favorably with the Blasius solution while avoiding the ill conditioning of the equations at very low Mach numbers[57]. Note that the higher order solutions are in closer agreement to the Blasius solution than the second order solution. The results shown in figure 19b represent a uniform refinement of the cases in figure 19a. At this level of refinement, all the schemes give visually similar distributions of the skin friction. Note that there is still a small oscillation in the skin friction at the outflow for all the cases, probably due to the combination of strong enforcement of the no-slip condition and weak enforcement of the 
outflow condition.

\subsubsection{NACA 0012 Airfoil}

Figure 20 shows a sample grid for a case of laminar flow over a NACA 0012 airfoil. The flow conditions are $M_{\infty}=0.8, \alpha=10^{\circ}$, and $R e=500$, corresponding to case A2 in [58]. This case represents a relatively severe test of the method since much of the upper surface flow is separated. In fact, on sufficiently fine grids, obtaining a steady solution was impossible, and to obtain the coarse grid solutions, the number of Krylov vectors used in the GMRES scheme had to be increased to 30. The cases shown were all run on a grid that began as a structured C-type mesh having 73 points in the circumferential direction and 25 points in the normal direction. This $\mathrm{C}$ mesh was used to distribute Lagrange points on a triangular finite-element mesh which was then converted to the required Bezier representation. Thus all the cases have the same number of degrees of freedom.

A comparison of skin friction distributions for each order of accuracy is presented in figure 21. Note that there are now significant changes among the different orders of accuracy up through fifth order $(n=4)$, particularly at the leading and trailing edges. This is to be expected since the calculations are on extremely coarse meshes. Because the skin friction is based on derivatives of the flow variables, it can be expected to converge one order less than the solution. In other words, a second-order solution should exhibit firstorder convergence of the skin friction.

An unexpected observation of particular importance can be seen in the convergence histories presented in figure 22. Note that contrary to conventional wisdom, the nonlinear system converges more quickly as the accuracy of the scheme is increased. Not reflected in the figure, however, is the fact that the linear system does become more difficult to solve. This may be due to the preconditioning - a diagonal preconditioner can be expected to degrade in performance as the matrix becomes less sparse. This difficulty does not seem to significantly impact convergence, however, since the GMRES algorithm meets the preset limits of 20 Krylov vectors and one restart early in the computation. The actual pro- 
cessing time is virtually the same for these cases. An analysis of the number of floating point operation count in a single inviscid residual evaluation indicates that for the same problem size (i.e. degrees of freedom), the third and fourth order schemes incur approximately 15 per cent fewer floating point operations than the second and fifth order schemes for each evaluation of the inviscid residual. 


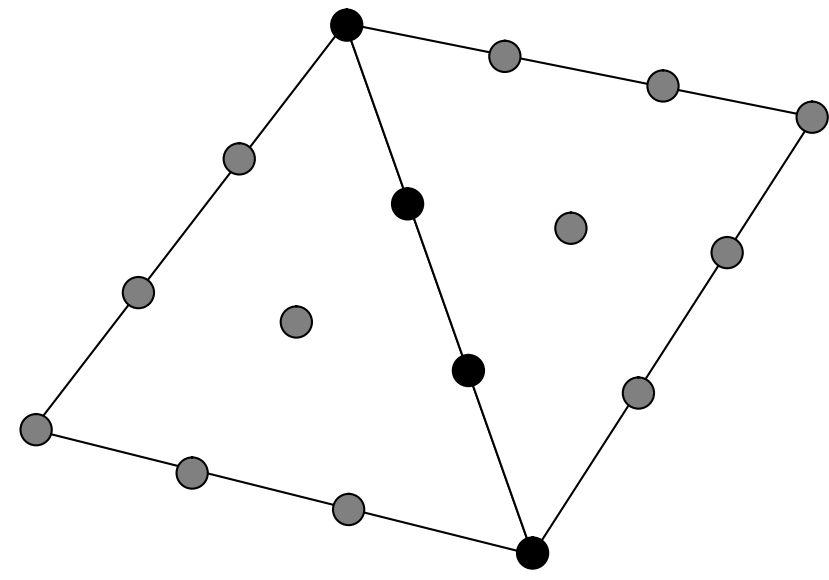

- shared points o control points

a) Enforcement of continuity via shared control points (cubic elements depicted).

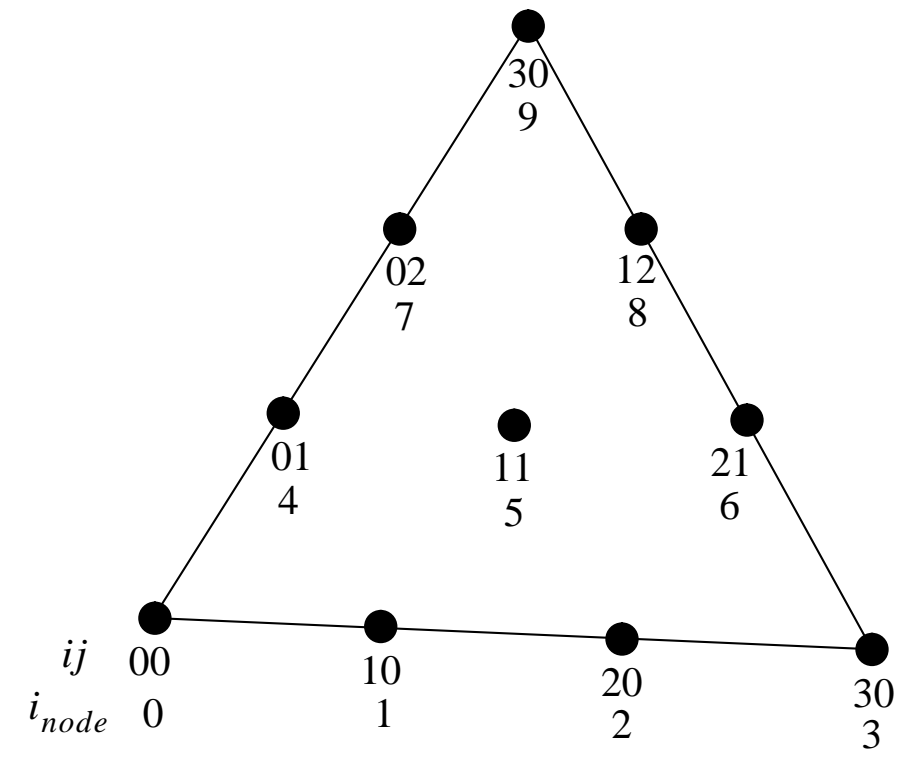

b) Indexing of control points within an element (cubic element depicted).

Figure 4. Element data distribution using triangular Bezier patches. 
- boundary control points o control points

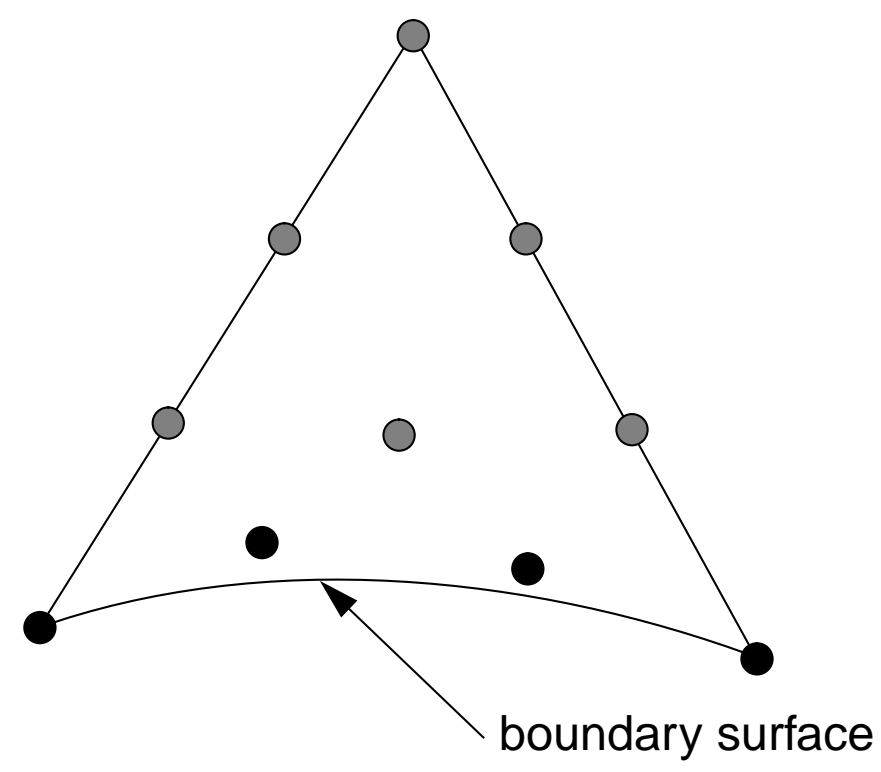

Figure 5. Boundary element data distribution using Bezier segments. 


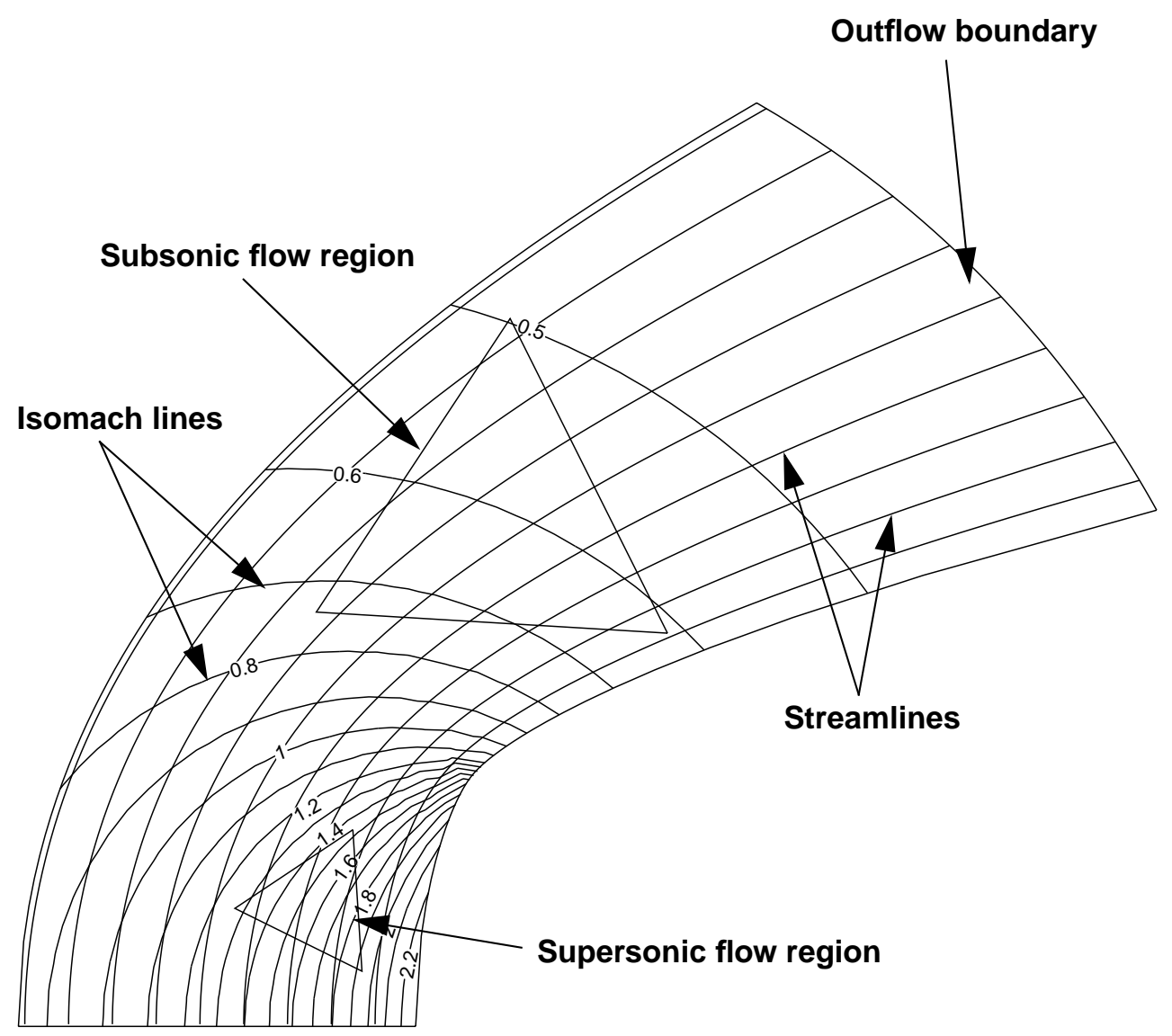

Figure 6. Computational regions in Ringleb flow domain used for analysis. 


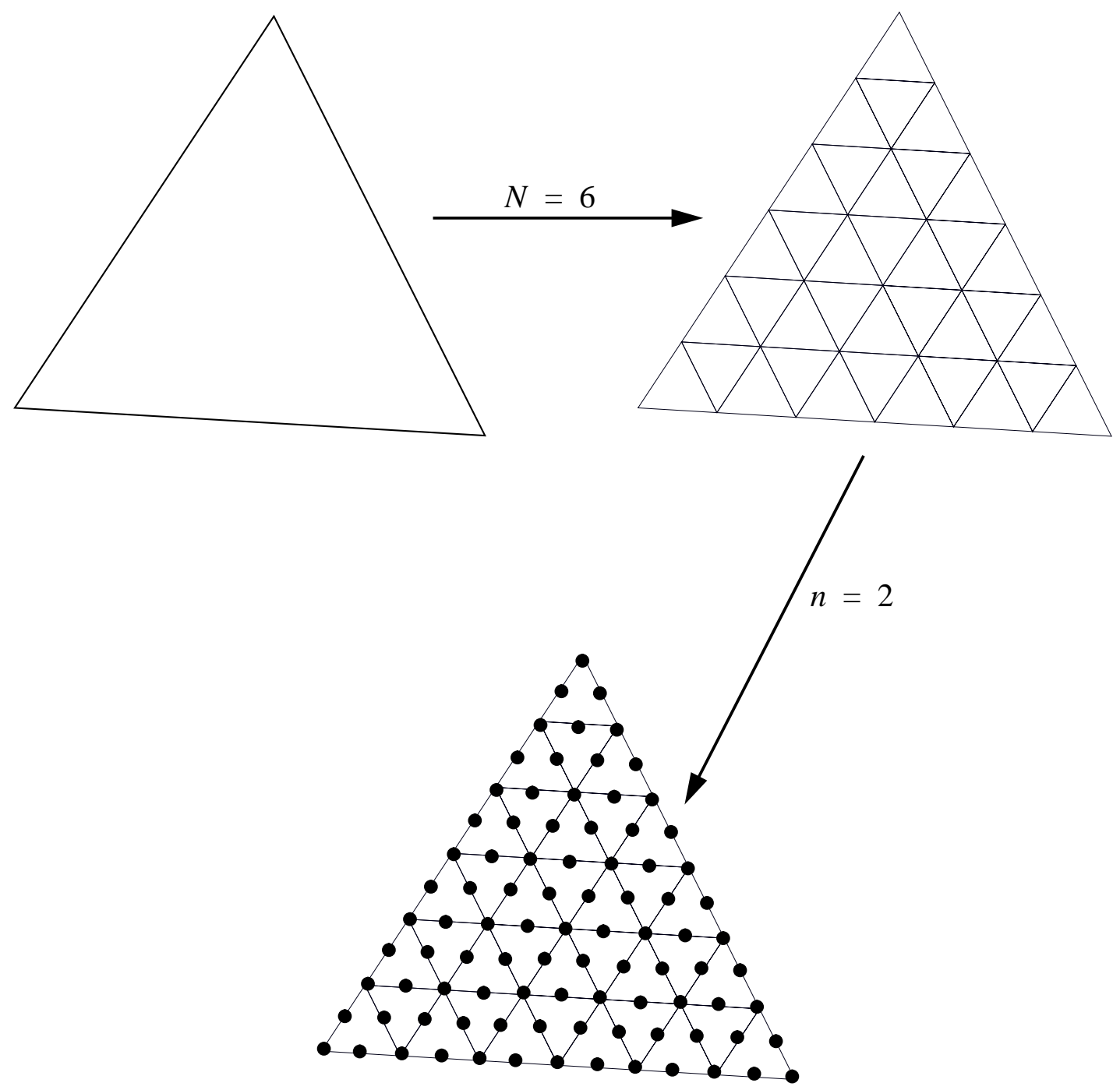

Figure 7. Generation of finite element meshes for Ringleb flow. 


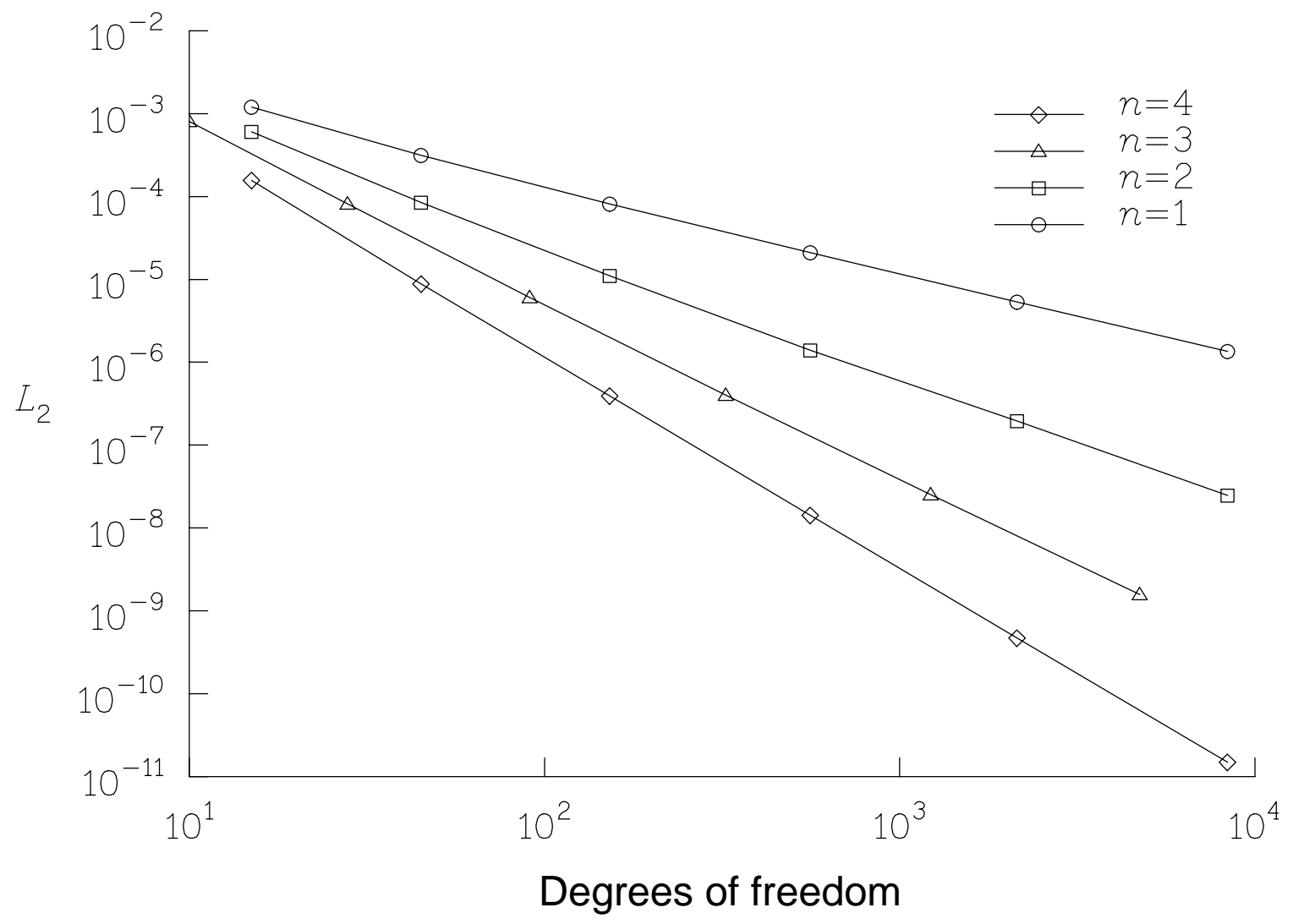

Figure 8. Error norms in subsonic region of Ringleb flow. 


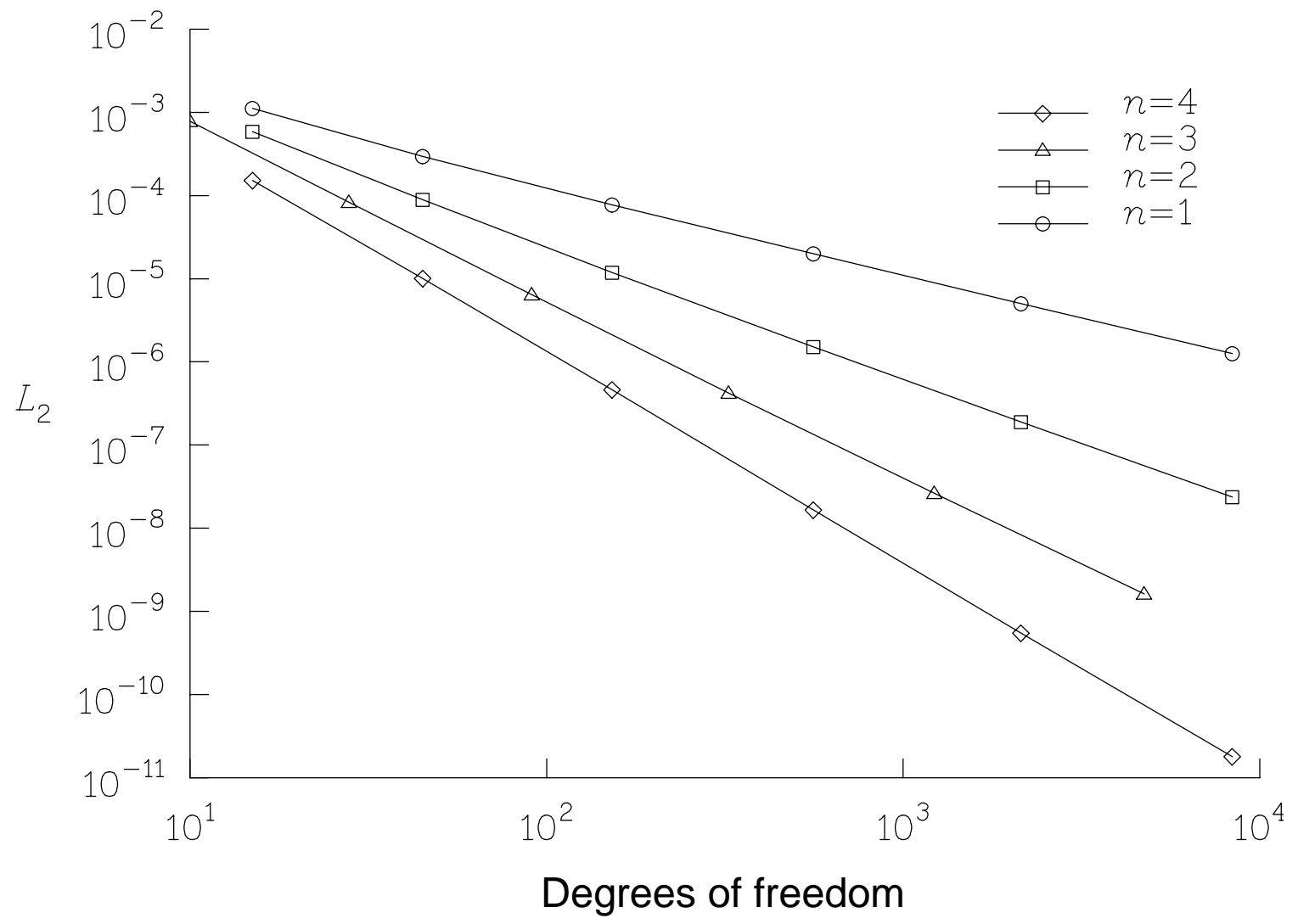

Figure 9. Error norms in supersonic region of Ringleb flow. 


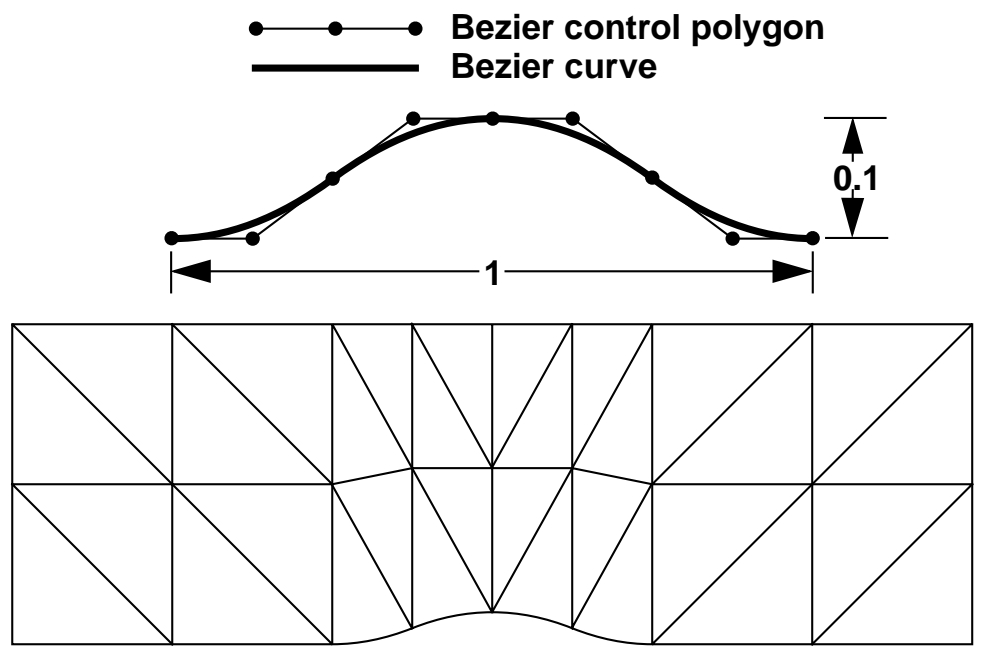

a) Geometry and baseline grid.

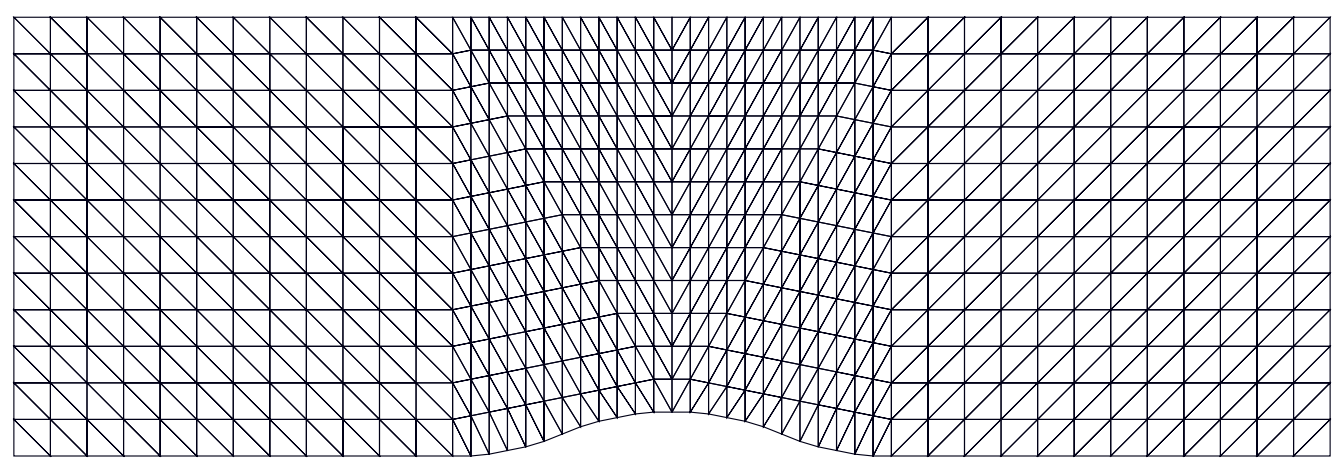

b) Sample grid, $N=2, n=3$.

Figure 10. Geometry and grid for parabolic bump in a channel. 


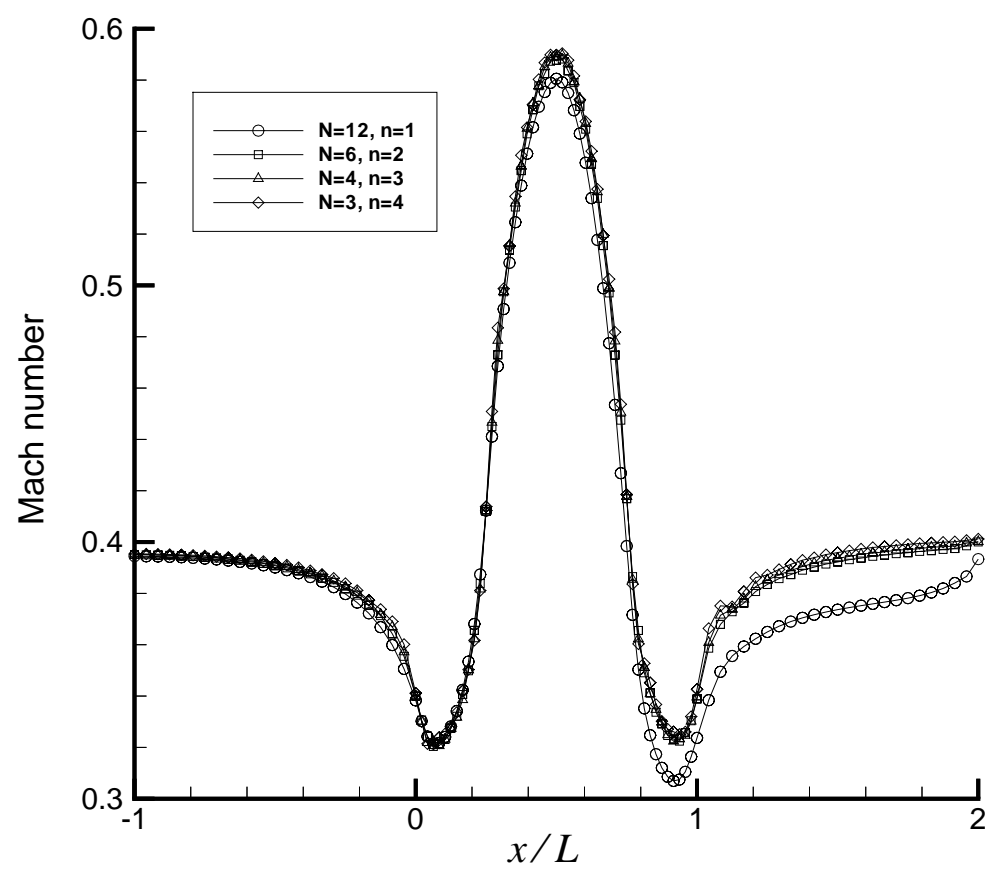

a) Comparison of high order SU/PG schemes.

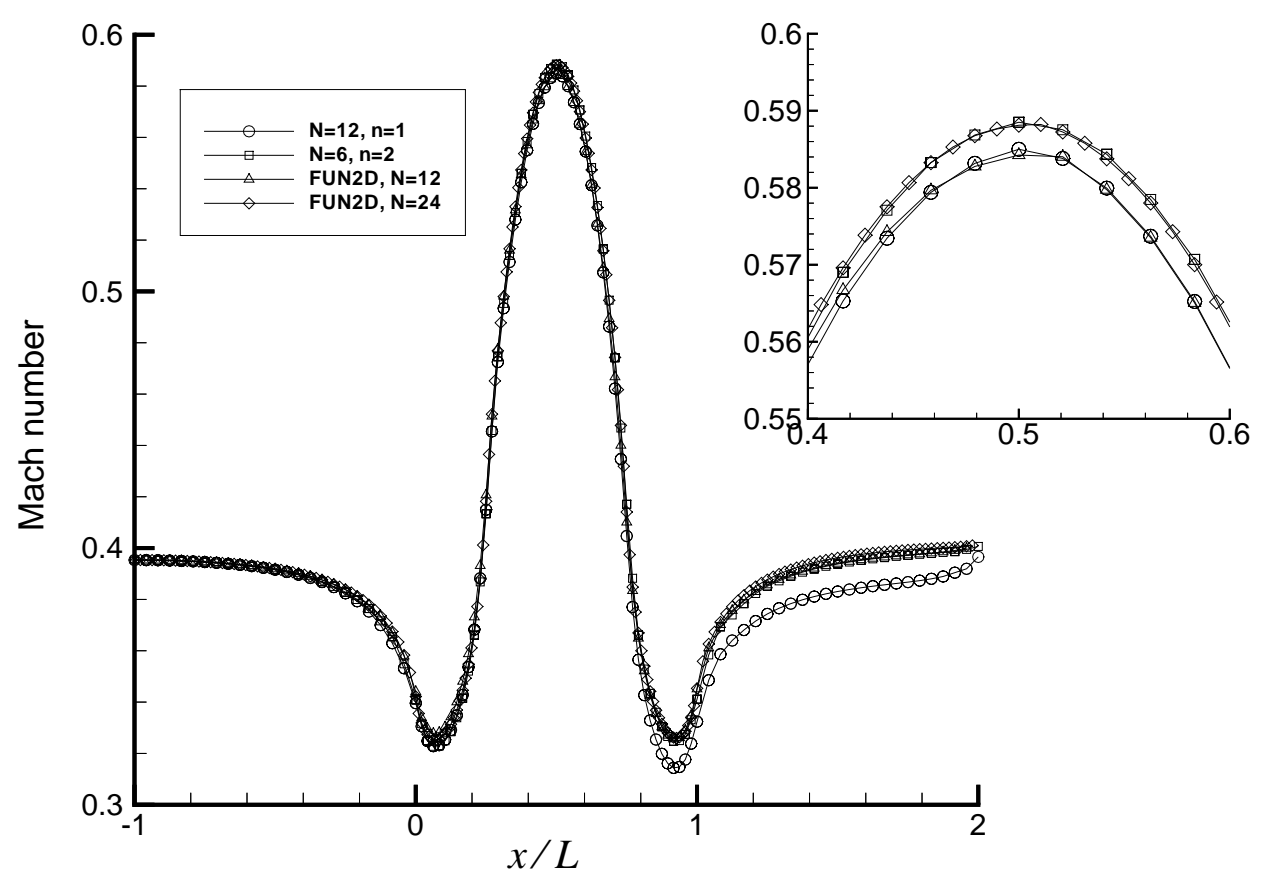

b) Comparison with second order finite volume scheme.

Figure 11. Surface Mach number distribution for parabolic bump in a channel. 


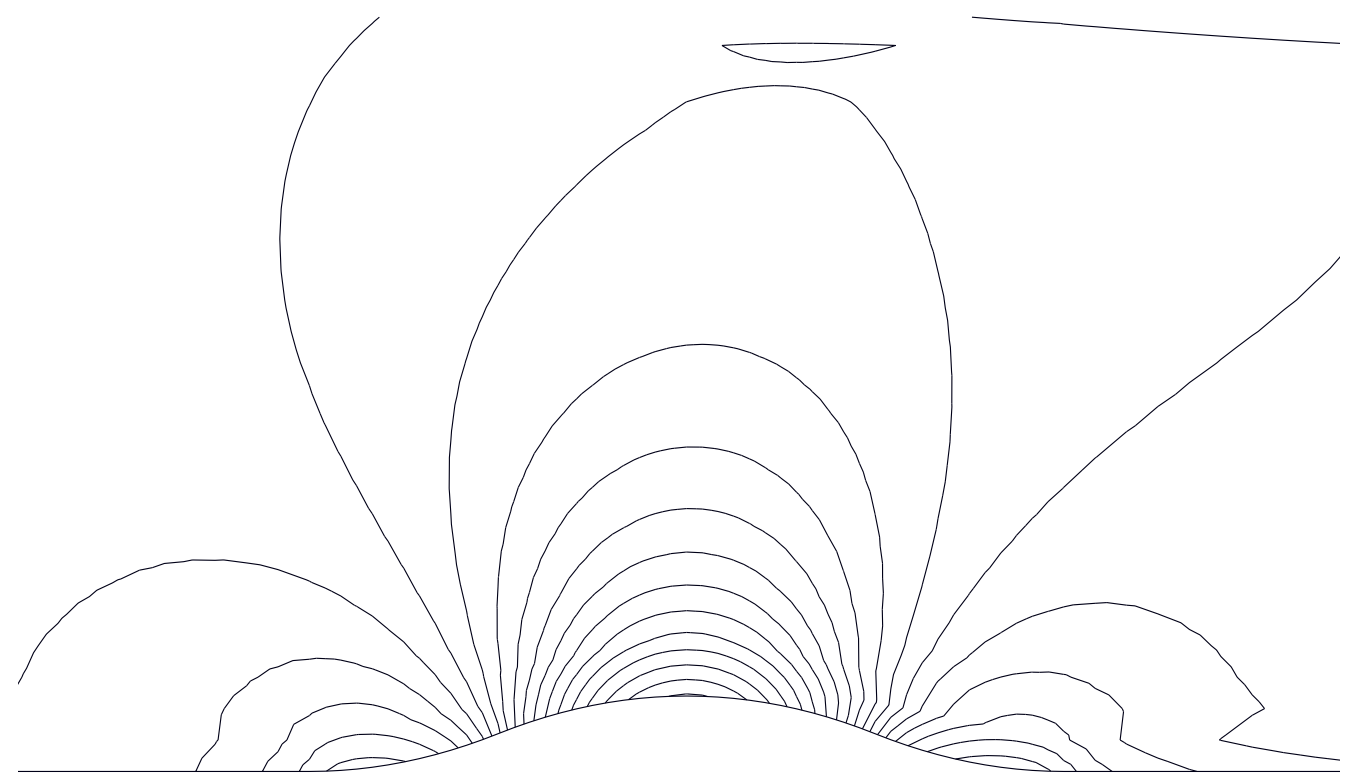

a) Second order SU/PG scheme.

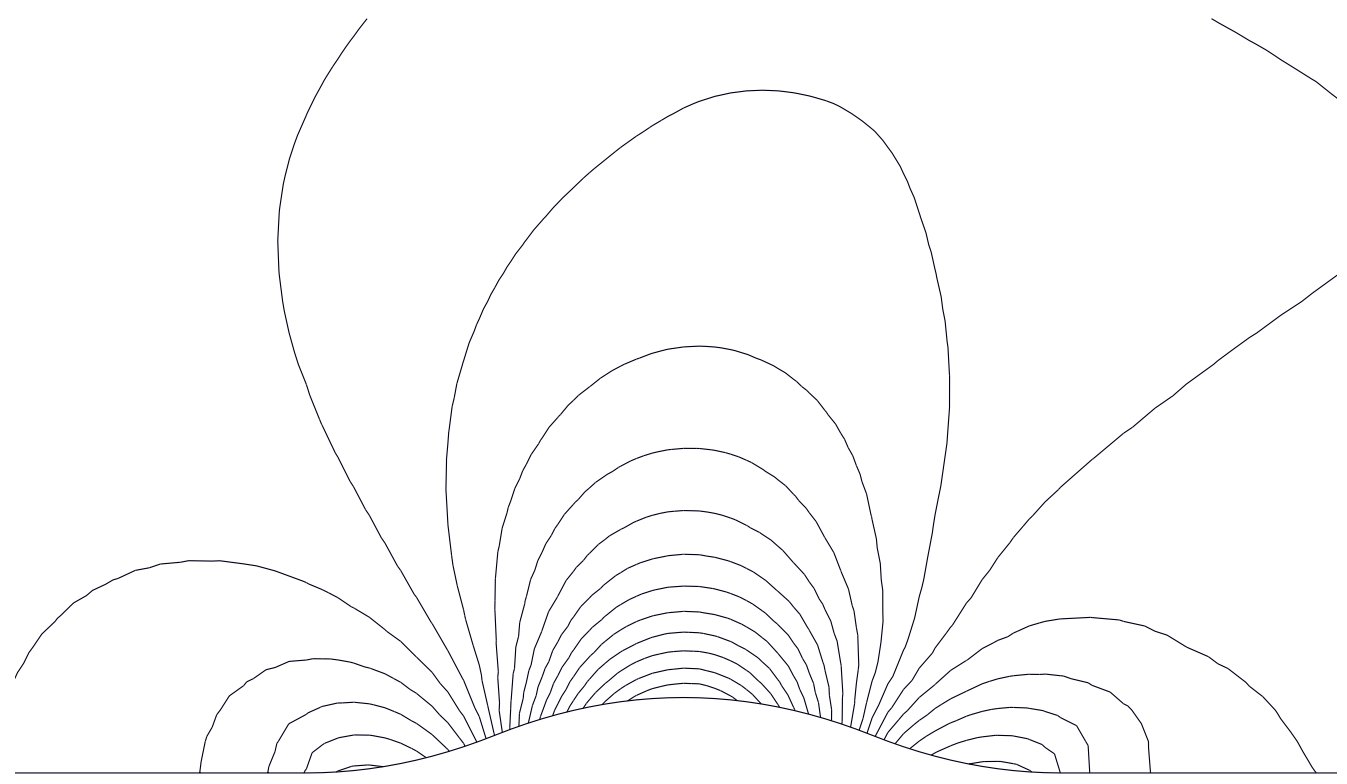

b) Second order finite volume scheme.

Figure 12. Mach number contours for parabolic bump on a wall. 


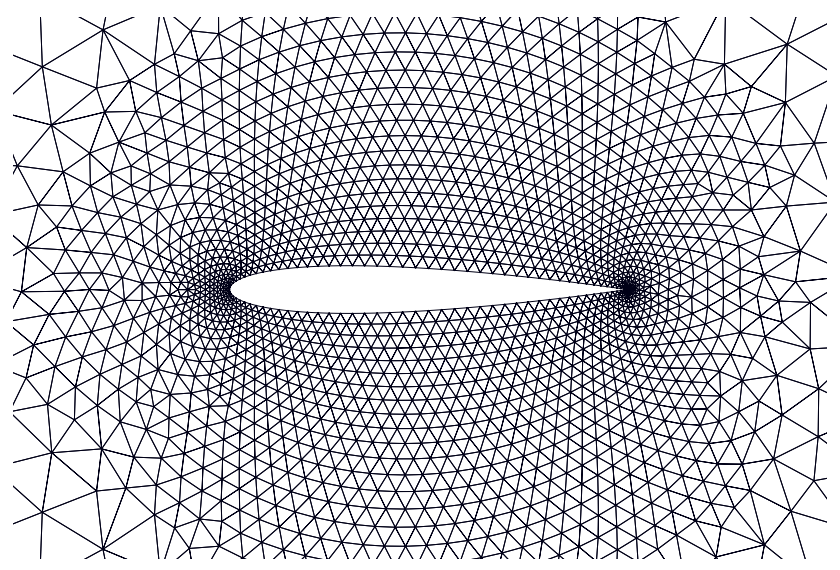

a) Fine grid.

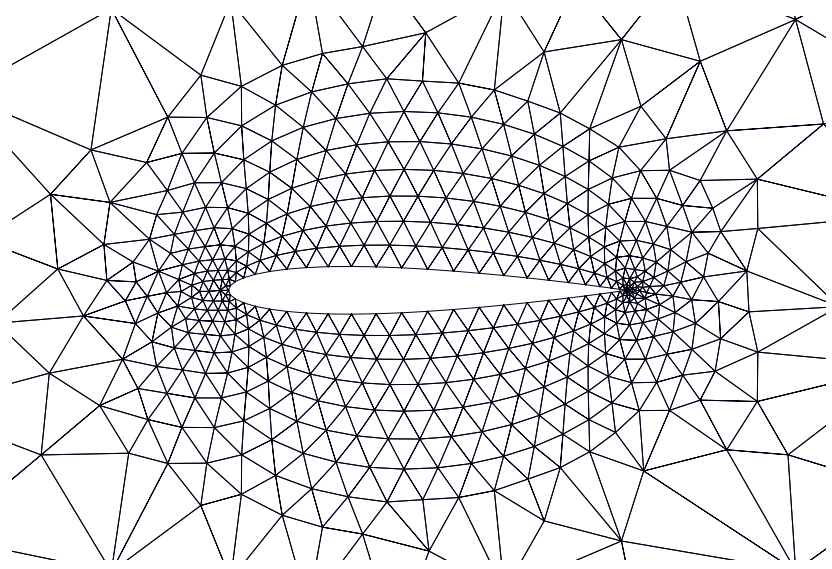

b) Medium grid.

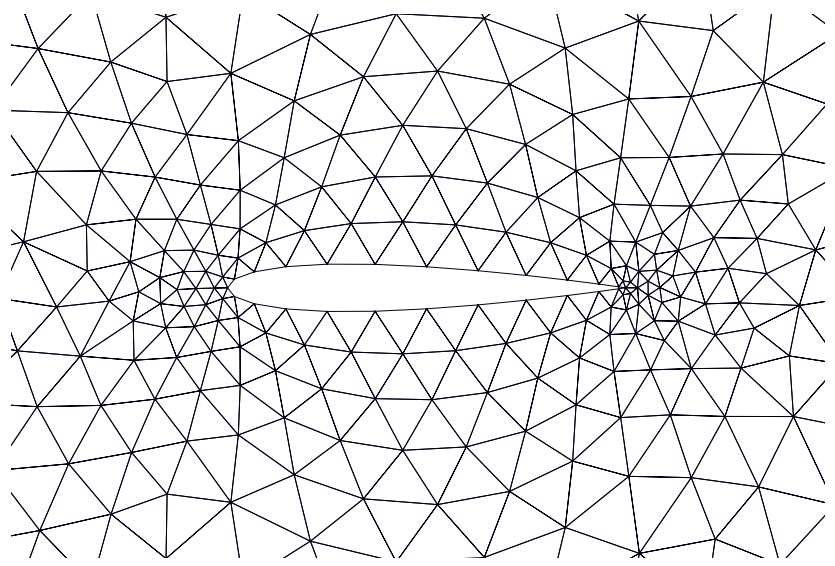

c) Coarse grid.

Figure 13. Fine grid for inviscid flow over a NACA 0012 airfoil. 


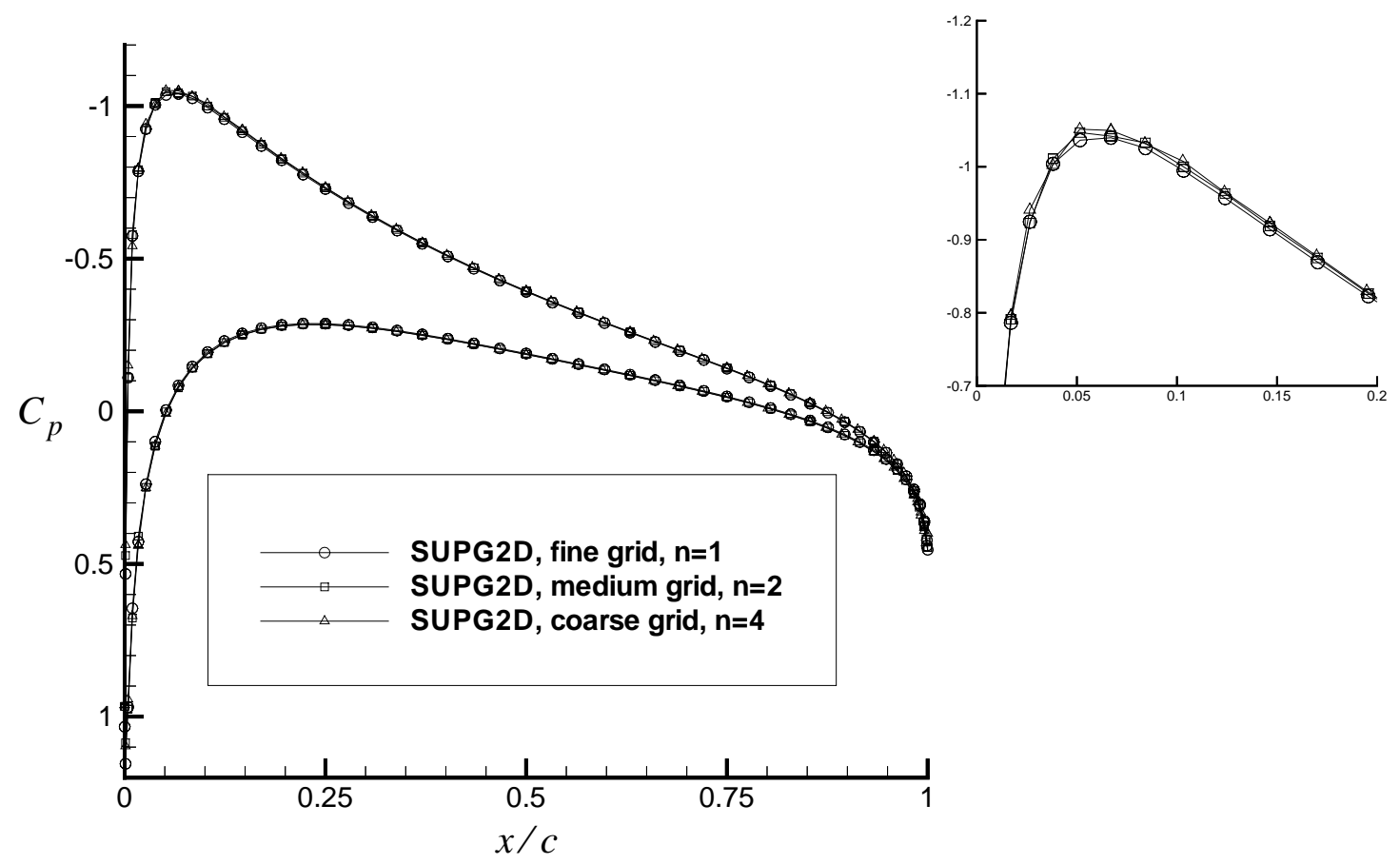

a) Comparison of high order SU/PG schemes.

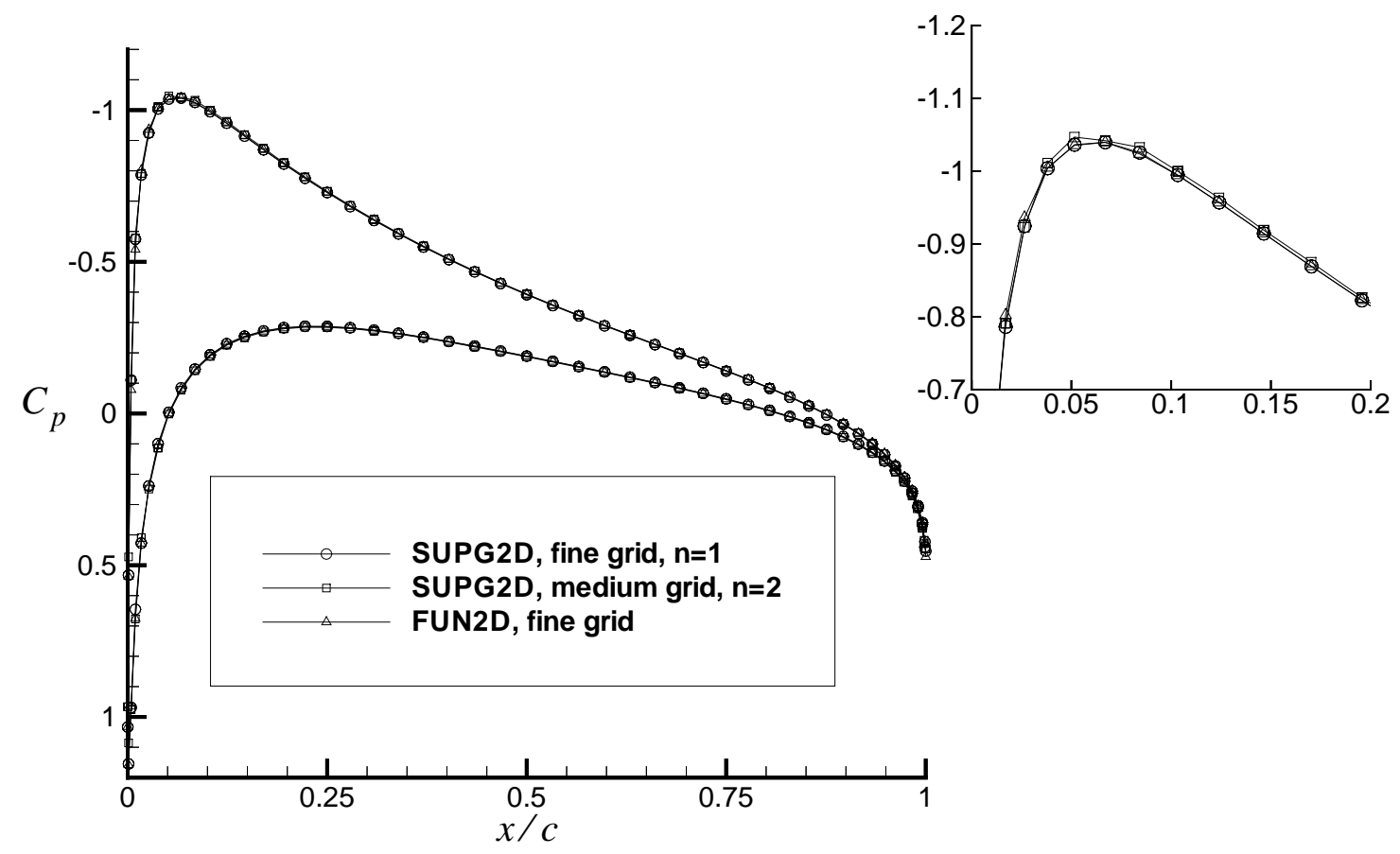

b) Comparison with second order finite volume scheme.

Figure 14. Surface pressure distribution for subcritical NACA 0012 airfoil. 


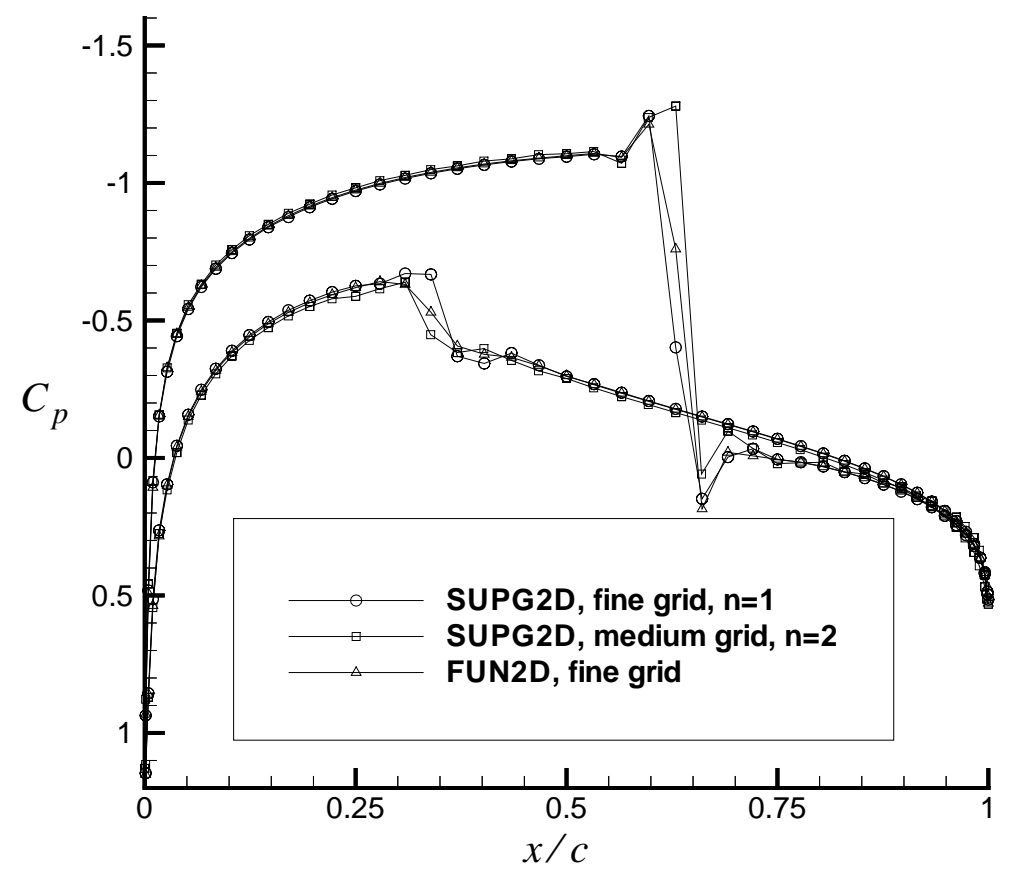

Figure 15. Surface pressure distribution for transonic NACA 0012 airfoil. 


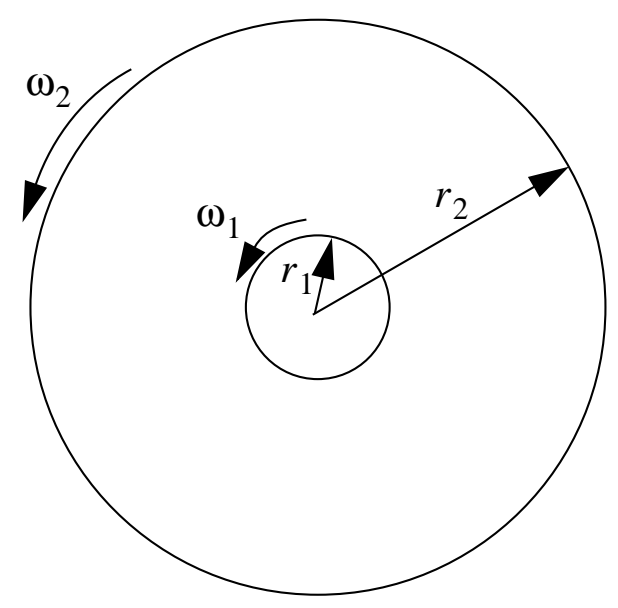

a) Geometry.

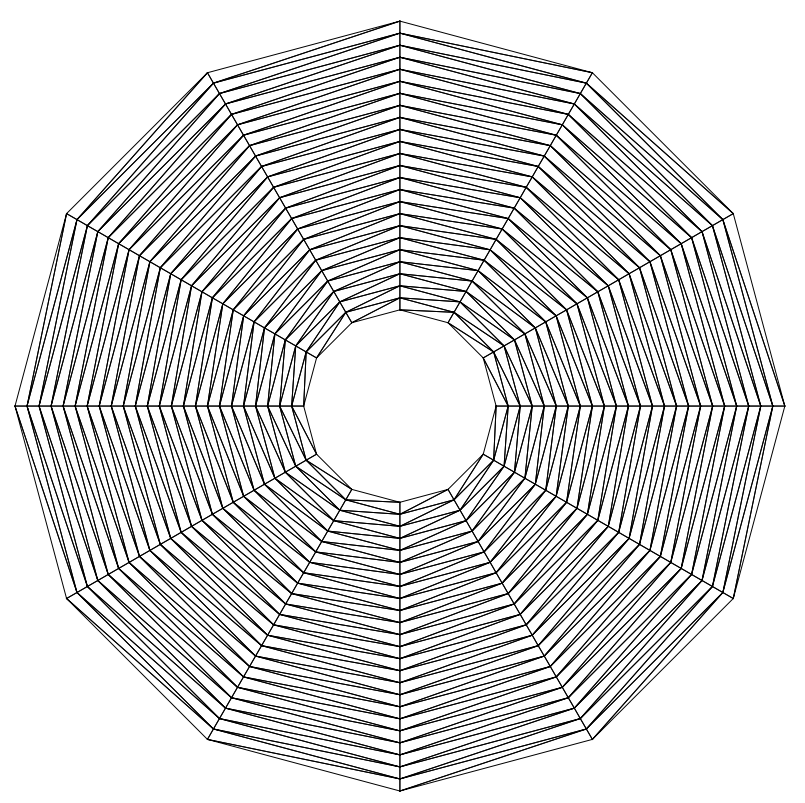

b) Sample grid.

Figure 16. Solution domain for rotational Couette flow. 


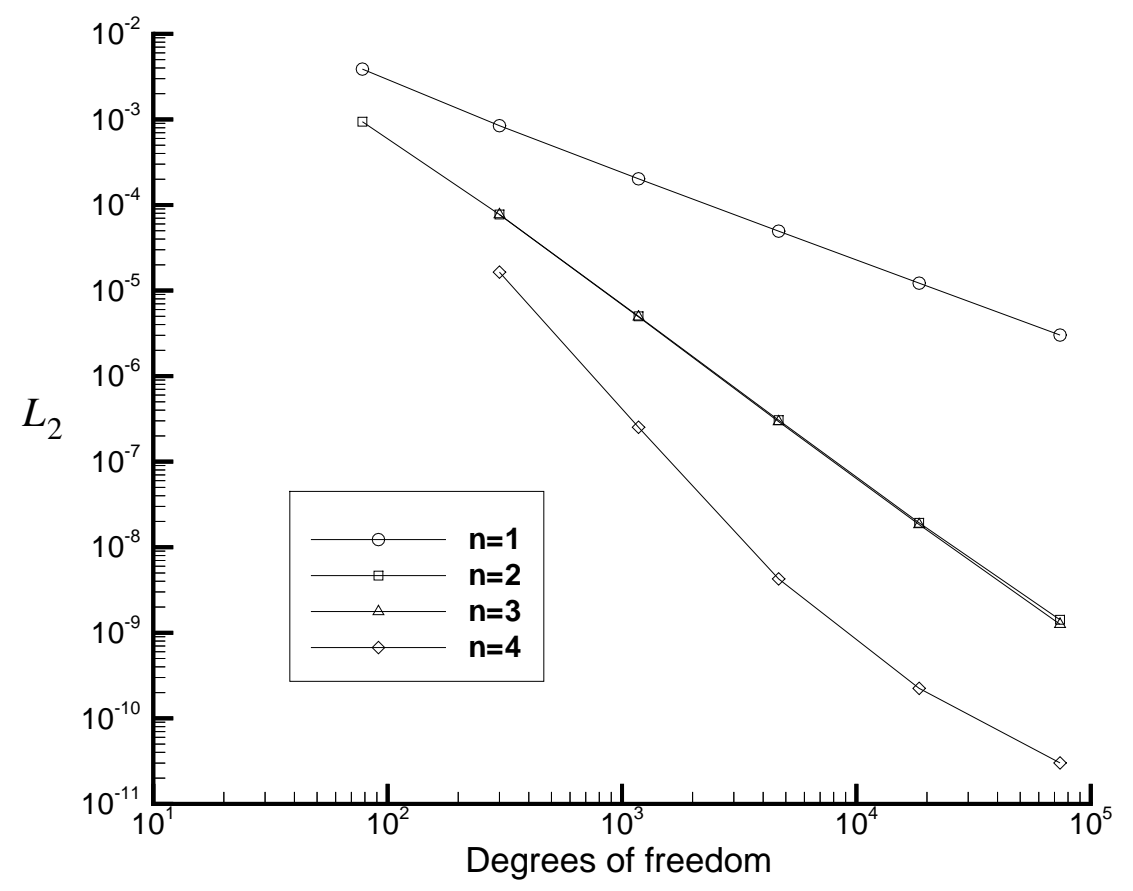

Figure 17. Error in circumferential velocity for rotational Couette flow. 


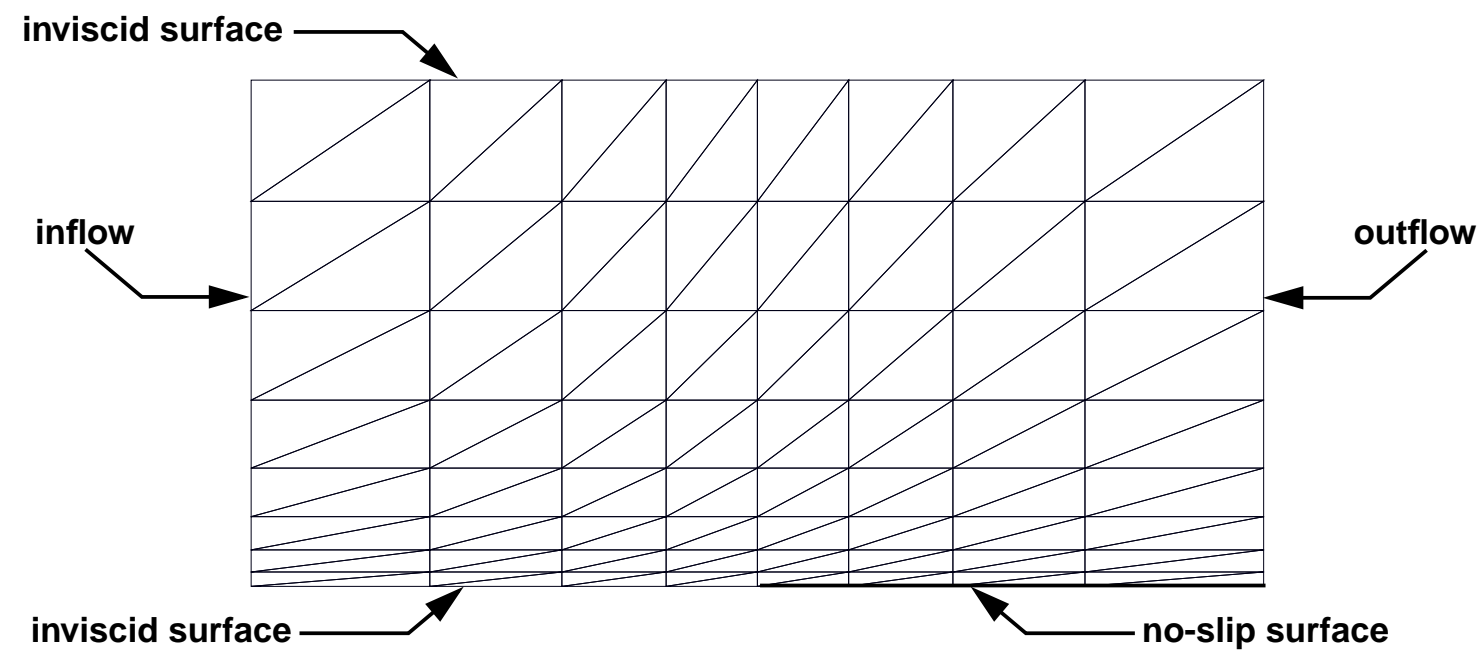

Figure 18. Solution domain and baseline grid for flat plate flow. 


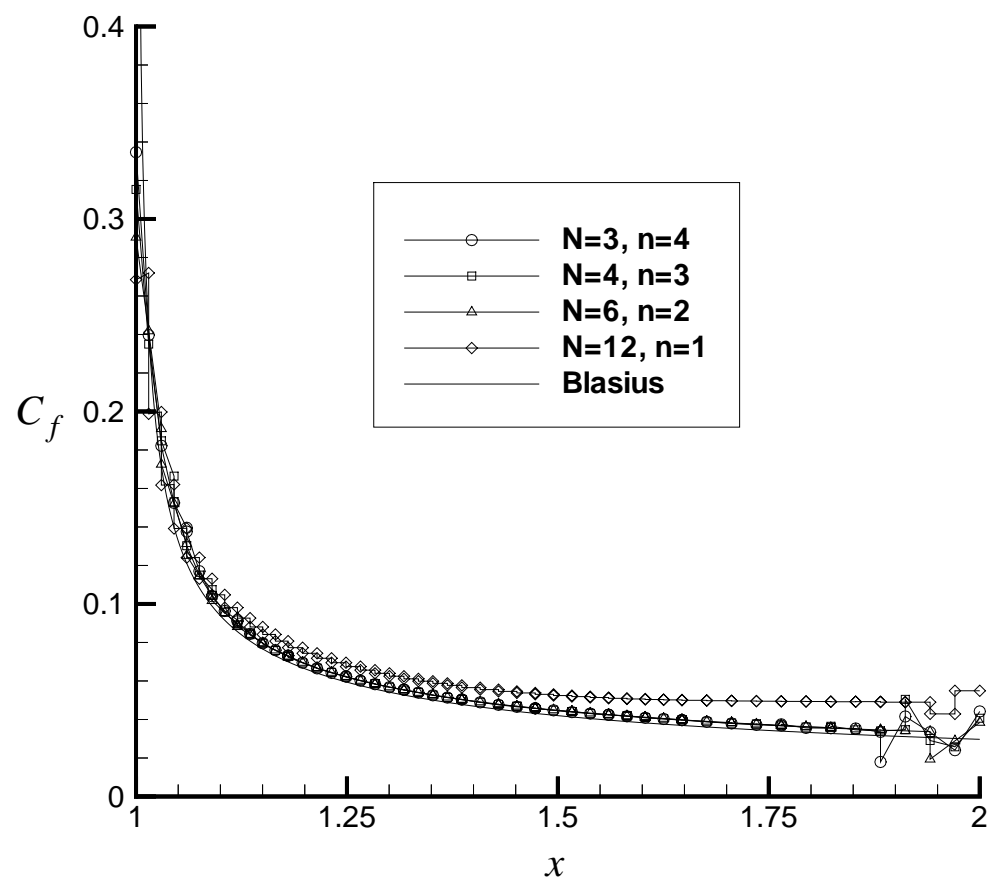

a) Medium grid.

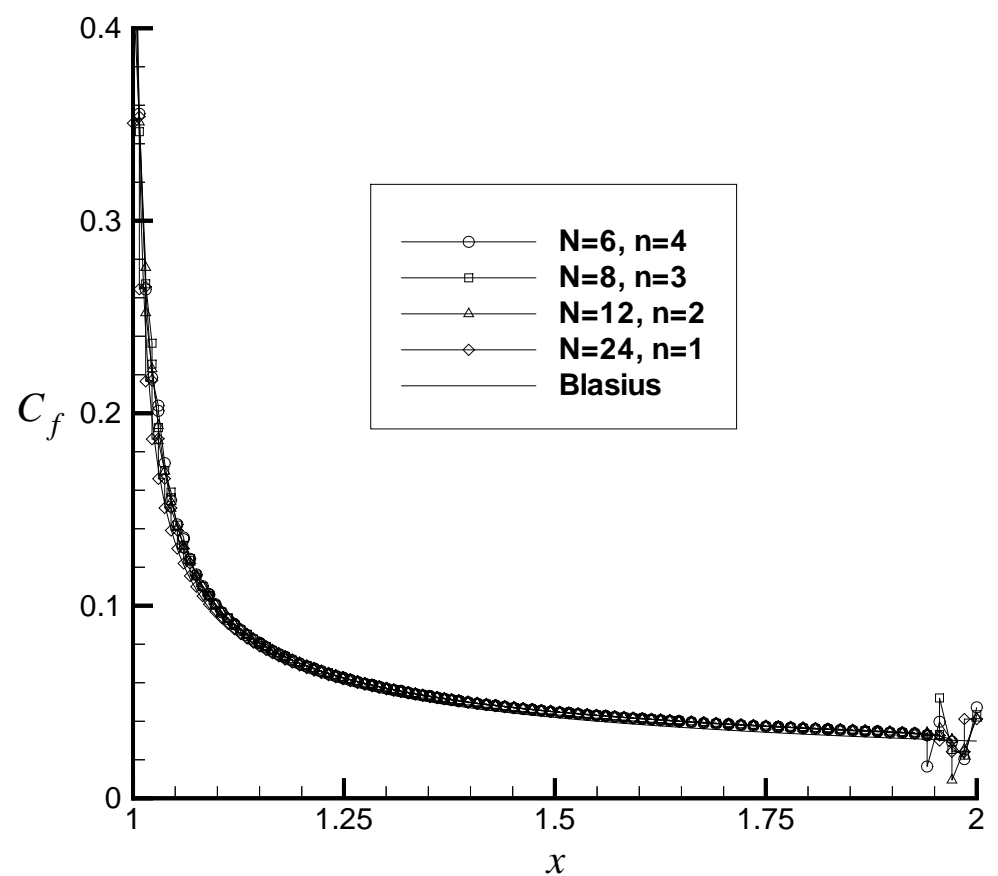

b) Fine grid.

Figure 19. Skin friction distributions for flat plate flow. 


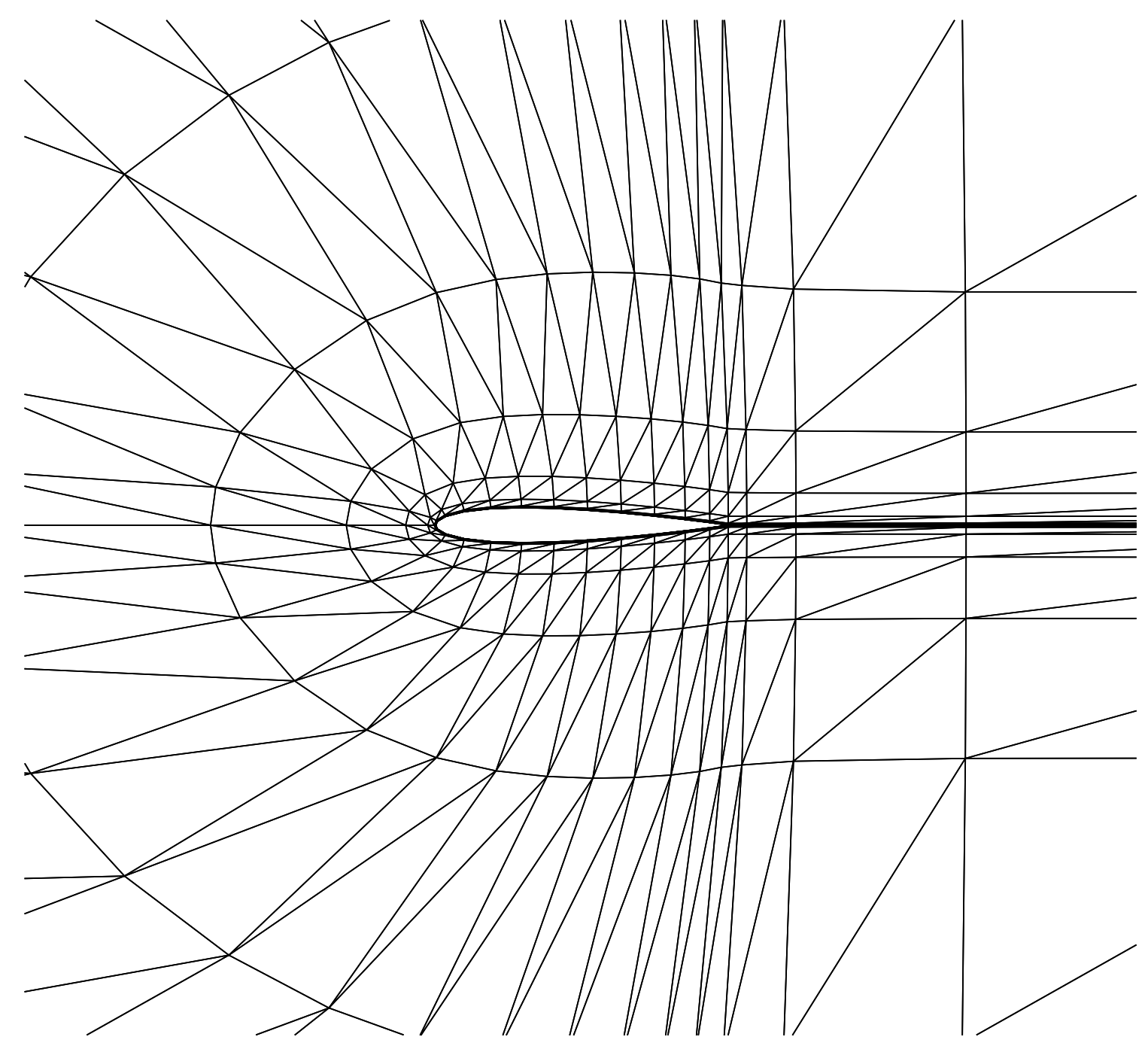

Figure 20. Sample grid for laminar flow over a NACA 0012 airfoil. 


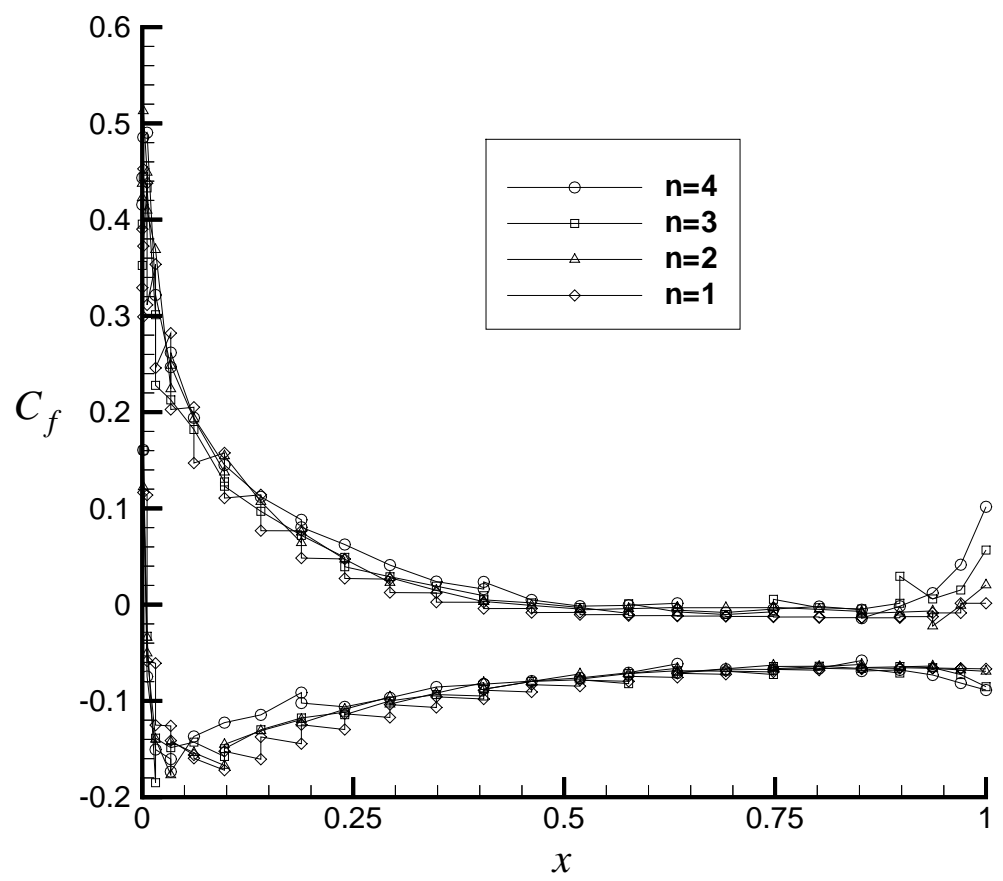

Figure 21. Skin friction distributions for laminar flow over a NACA 0012 airfoil. 


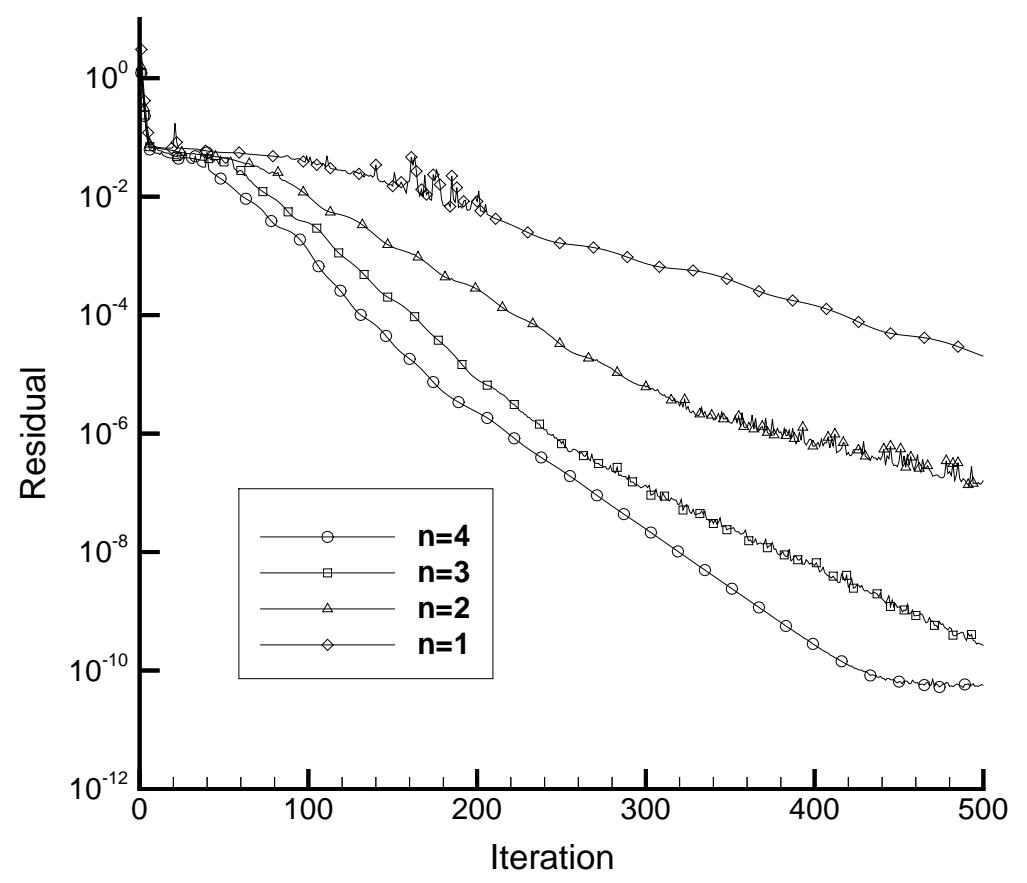

Figure 22. Convergence histories for laminar flow over a NACA 0012 airfoil. 


\section{Chapter 5}

\section{Concluding Remarks}

The SU/PG method has been applied for the first time to higher-order finite-element discretizations in one and two dimensions. Several test cases involving both subsonic and transonic inviscid flows in one and two dimensions were investigated as well as several subsonic laminar flow cases in two dimensions. High order accuracy was confirmed up to ninth order for one-dimensional flows and up to fifth order for two dimensional inviscid and laminar flows. The higher order schemes were found to provide significantly more accurate results using fewer computational resources. An appendix is included in which the method is applied to three-dimensional inviscid flows. Several important observations were made based on the results:

1. No modification of the SU/PG scheme is required to achieve high order accuracy. This essentially means that the order property of the scheme is independent of the formulation of the SU/PG parameter $\tau$ provided the original design criteria are met(i.e. that $\tau$ vanishes as the mesh is refined). There are indications from comparisons with a second order finite volume scheme, that an improved formulation of the $\tau$ matrix could further reduce error levels by a constant factor.

2. Significant improvement of the solution results from using the higher order schemes. Error norms for Ringleb flow and Couette flow indicate that the high order schemes outperform the second order scheme even on very coarse meshes. Analysis of operation counts indicates that based on problem size, the third and fourth order schemes 
are cheaper to compute than the second order scheme, and that the operation count of the fifth order scheme is comparable to the second order scheme.

3. Neglecting the Petrov-Galerkin contribution of the viscous terms in the two-dimensional schemes had no impact on the order properties of the scheme. In spite of the absence of these terms, the error norms for Couette flow converged at high order. There is the possibility, however, that inclusion of these terms could influence error levels by a constant factor.

4. The nonlinear system converges more quickly as the order of accuracy is increased for the same number of degrees of freedom. The linear system converges more slowly, but processing time is only slightly affected because the linear solver reaches the limit of its allotted resources per iteration early in the computation. This is contrary to popular belief that higher-order schemes suffer from convergence problems.

Clearly, the results shown here indicate that higher order schemes can greatly reduce the computational resources required to compute steady-state aerodynamics of complex configurations. In order to realize this potential, however, several areas must be explored in future research.

The primary difficulty in using the higher-order schemes was the generation of suitable higher-order discretizations of the flow domain when curved boundaries were involved. No general solution was found - each case required its own preprocessor to generate the higher-order finite-element mesh from an existing structured mesh or linear finite-element mesh. Particularly difficult to generate are meshes that are highly stretched to compute viscous flows - simply moving the boundary control points to match the surface creates overlapping elements which are unacceptable to the scheme. These problems are further compounded in three dimensions. To facilitate application of the higher-order schemes in general, grid generation techniques must be adapted to handle high-order discretizations and must be more closely tied to geometry definitions (e.g. CAD systems). This remains as an avenue of future work.

Other issues remaining for future research with the two-dimensional scheme are improvement of the Petrov-Galerkin parameter $\tau$ as discussed in chapter 2 and the incor- 
poration of the Petrov-Galerkin contribution of the viscous terms, both of which may further reduce error levels by a constant factor. The GMRES algorithm with block-diagonal preconditioning provided adequate convergence of the linear system, but an improved preconditioning scheme could bring gains in efficiency by allowing a larger pseudotime step. The addition of a turbulence model will allow computation of high Reynolds number flows. The three-dimensional scheme (presented in appendix A) can benefit from these same improvements and will also require the addition of viscous terms. 


\section{References}

1. Barth, T. J. and Jesperson, D. C.; "The Design and Application of Upwind Schemes on Unstructured Meshes," AIAA 27th Aerospace Sciences Meeting, AIAA 89-0366, January 1989.

2. Whitaker, D. L., Slack, D. C., and Walters, R. W.; "Solution Algorithms for the TwoDimensional Euler Equations on Unstructured Meshes," AIAA 90-0697, 1990.

3. Jameson, A., Baker, T., and Weatherill, N.; "Calculation of Inviscid Transonic Flow over a Complete Aircraft," AIAA 24th Aerospace Sciences Meeting, AIAA 86-0103, January 1986.

4. Jameson, A. and Baker, T.; "Improvements to the Aircraft Euler Method," AIAA 25th Aerospace Sciences Meeting, AIAA 87-0452, January 1987.

5. Jameson, A. and Mavriplis, D.; "Finite Volume Solution of the Two-Dimensional Euler Equations on a Regular Triangular Mesh," AIAA 23rd Aerospace Sciences Meeting, AIAA 85-0435, January 1985.

6. Mavriplis, D. and Jameson, A.; "Multigrid Solution of the Navier-Stokes Equations on Triangular Meshes," AIAA Journal, vol. 28, pp. 1415-1425, January 1986.

7. Barth, T. J.; "Numerical Aspects of Computing Viscous High Reynolds Number Flows on Unstructured Meshes," AIAA 29th Aerospace Sciences Meeting, AIAA 91-0721, January 1991.

8. Barth, T. J. and Frederickson, P. O.; "Higher Order Solution of the Euler Equations on Unstructured Grids Using Quadratic Reconstruction,” AIAA 90-0013, January 1990.

9. Hu, C. and Shu, C. W.; "Weighted Essentially Non-Oscillatory Schemes on Triangular 
Meshes," NASA CR-1998-208459, July 1998.

10. Halt, D. W.; “A Compact High-Order Euler Solver for Unstructured Grids,” Ph.D. dissertation, Washington University, May 1992.

11. Halt, D. W. and Agarwal, R. K.; "A Compact Higher Order Characteristic-Based Euler Solver for Unstructured Grids," AIAA 29th Applied Aerodynamics Conference, AIAA 91-3234, September 1991.

12. Zienkiewicz, O. C.; The Finite Element Method, McGraw-Hill Book Company (UK) Limited, London, 1977.

13. Hughes, T. J. R.; The Finite Element Method: Linear Static and Dynamic Finite Element Analysis, Prentice-Hall, Inc., New Jersey, 1987.

14. Baker, A. J. and Pepper, D. W.; Finite Elements 1-2-3, McGraw-Hill, Inc., New York, 1991.

15. Cockburn, B. and Shu, C.-W.; “TVB Runge-Kutta Local Projection Discontinuous Galerkin Finite Element Method for Conservation Laws II: General Framework," Mathematics of Computation, vol. 52, pp. 411-435, 1989.

16. Cockburn, B., Lin, S. Y., and Shu, C.-W.; "TVB Runge-Kutta Local Projection Discontinuous Galerkin Finite Element Method for Conservation Laws III: One Dimensional Systems," Journal of Computational Physics, vol. 84, pp. 90-113, 1989.

17. Cockburn, B. Hou, S., and Shu, C.-W.; “TVB Runge-Kutta Local Projection Discontinuous Galerkin Finite Element Method for Conservation Laws IV: The Multidimensional Case," Mathematics of Computation, vol. 54, pp. 545-581, 1990.

18. Bey, K. S.; “An HP-Adaptive Discontinuous Galerkin Method for Hyperbolic Conservation Laws," Ph.D. dissertation, Univ. of Texas at Austin, 1994.

19. Atkins, H. L. and Shu, C.-W.; "Quadrature-Free Implementation of the Discontinuous Galerkin Method for Hyperbolic Equations," 2nd AIAA/CEAS Aeroacoustics Conference, AIAA 96-1683, May 1996.

20. Lowrie, R. B., Roe, P. L. and van Leer, B.; “A Space-Time Discontinuous Galerkin Method for the Time-Accurate Numerical Solution of Hyperbolic Conservation Laws," AIAA 95-1658, 1995. 
21. Bey, K. S. and Oden, J. T.; “A Runge-Kutta Discontinuous Finite Element Method for High Speed Flows," AIAA 10th Computational Fluid Dynamics Conference, AIAA 911575-CP, June 1991.

22. Bassi, F. and Rebay, S.; "Discontinuous Finite Element High Order Accurate Numerical Solution of the Compressible Navier-Stokes Equations,' ICFD Conference on Numerical Methods in Fluid Dynamics, Bangalor, India, July 1994.

23. Bassi, F. and Rebay, S.; “Accurate 2D Euler Computations by Means of a High-Order Discontinuous Finite Element Method," Proceedings of the 14th International Conference on Numerical Methods in Fluid Dynamics, Oxford, England, April 1995.

24. Brooks, A. N. and Hughes, T. J. R.; "Streamline Upwind/Petrov-Galerkin Formulations for Convection Dominated Flows with Particular Emphasis on the Incompressible Navier-Stokes Equations," Computer Methods in Applied Mechanics and Engineering, vol. 32, pp. 199-259, 1982.

25. Brezzi, F., Franca, L. P., Hughes, T. J. R., and Russa, A.; "Stabilization Techniques and Subgrid Scales Capturing," The State of the Art in Numerical Analysis, based on the proceedings of the Conference on the State of the Art in Numerical Analysis, York, England, April 1996, IMA Conference Series, Vol. 63 (I.S. Duff and G.A. Watson, eds.), Oxford University Press, pp. 391-406.

26. Hughes, T. J. R. and Mallet, M.; "A New Finite Element Formulation for Computational Fluid Dynamics: III. The Generalized Streamline Operator for Multidimensional Advective-Diffusive Systems," Computer Methods in Applied Mechanics and Engineering, vol. 58, pp. 305-328, 1986.

27. Hughes, T. J. R. and Mallet, M.; “A New Finite Element Formulation for Computational Fluid Dynamics: IV. A Discontinuity-Capturing Operator for Multidimensional Advective-Diffusive Systems," Computer Methods in Applied Mechanics and Engineering, vol. 58, pp. 329-336, 1986.

28. Hughes, T. J. R., Franca, L. P., and Hulbert, G. M.; “A New Finite Element Formulation for Computational Fluid Dynamics: VIII. The Galerkin/Least-Squares Method for Advective-Diffusive Equations," Computer Methods in Applied Mechanics and Engineer- 
ing, vol. 73, pp. 173-189, 1989.

29. Soulaïmani, A. and Fortin, M.; "Finite element solution of compressible viscous flows using conservative variables," Computer Methods in Applied Mechanics and Engineering, No. 118, pp. 319-350, 1994.

30. Franca, L. P., Harari, I., Hughes, T. J. R., Mallet, M., Shakib, F., Spelce, T. E., Chalot, F., and Tezduyar, T. E.; "A Petrov-Galerkin Finite Element Method for the Compressible Euler and Navier-Stokes Equations," Numerical Methods for Compressible Flows - Finite Difference, Element and Volume Techniques; Proceedings of the Winter Annual Meeting, American Society of Mechanical Engineers, New York, pp. 19-43, 1986.

31. Brueckner, F. P. and Heinrich, J. C.; "Petrov-Galerkin Finite Element Method for Compressible Flows," International Journal for Numerical Methods in Engineering, Vol. 32, pp. 255-274, 1991.

32. Carette, J.-C., Deconinck, H., Paillere, H., and Roe, P. L.; "Multidimensional Upwinding: Its Relation to Finite Elements," International Journal for Numerical Methods in Fluids, Vol. 20, pp. 935-955, 1995.

33. Paillere, H., Carette, J.-C., and Deconinck, H.; "Multidimensional Upwind and SUPG Methods for the Solution of the Compressible Flow Equations on Unstructured Grids," in von Karman Institute for Fluid Dynamics Lecture Series 1994-05: Computational Fluid Dynamics, von Karman Institute for Fluid Dynamics, Rhode Saint Genese, Belgium, 1994.

34. Shakib, F.; "Finite Element Analysis of the Compressible Euler and Navier-Stokes Equations," Ph.D. dissertation, Stanford University, August 1988.

35. Shakib, F., Hughes, T. J. R., and Johan, Z; A New Finite Element Formulation for Computational Fluid Dynamics: X. The Compressible Euler and Navier-Stokes Equations," Computer Methods in Applied Mechanics and Engineering, vol. 89, pp. 141-219, 1991.

36. Chiocchia, G.; "Exact Solution to Transonic and Supersonic Flows," Test Cases for Inviscid Flow Field Methods, AGARD Advisory Report AR-211, May 1985.

37. Chossat, P and Iooss, G.; The Couette-Taylor Problem, Springer-Verlag New York, 
Inc., 1994.

38. Courant, R., Isaacson, E., and Reeves, M.; "On the Solution of Nonlinear Hyperbolic Differential Equations by Finite Differences," Comm. Pure and Applied Mathematics, vol. 5, pp. 243-255.

39. Hughes, T. J. R.; “Multiscale Phenomena: Green's Functions, the Dirichlet-to-Neumann Formulation, Subgrid Scale Models, Bubbles and the Origins of Stabilized Methods," Computer Methods in Applied Mechanics and Engineering, vol. 127, pp. 387-401, 1995.

40. Mizukami, A. and Hughes, T. J. R.; "A Petrov-Galerkin Finite Element Method for Convection-Dominated Flows: An Accurate Upwinding Technique for Satisfying the Maximum Principle," Computer Methods in Applied Mechanics and Engineering, vol. 50, pp. 181-193, 1985.

41. Casper, J and Carpenter, M. H.; "Computational Considerations for the Simulation of Shock-Induced Sound," NASA TM-110222, December 1995.

42. Ames Research Center Staff; "Equations, Tables, and Charts for Compressible Flow," NACA Report 1135, 1953.

43. Jansen, K. E., Collis, S. S., Whiting, C. and Shakib, F.; “A Better Consistency for LowOrder Stabilized Finite Element Methods," Computer Methods in Applied Mechanics and Engineering, to appear.

44. Cowper, G. R.; “Gaussian Quadrature Formulas for Triangles,” International Journal of Numerical Methods in Engineering, vol. 7, pp. 405-408, 1973.

45. Saad, Y., and Schultz, M. H.; "GMRES: A Generalized Minimum Residual Algorithm for Solving Nonsymmetric Linear Systems," SIAM Journal of Scientific and Statistical Computing, vol. 7, pp. 856-869, 1986.

46. Saad, Y.; "Krylov Subspace Techniques, Conjugate Gradients, Preconditioning and Sparse Matrix Solvers," in von Karman Institute for Fluid Dynamics Lecture Series 199405: Computational Fluid Dynamics, von Karman Institute for Fluid Dynamics, Rhode Saint Genese, Belgium, 1994.

47. McHugh, P. R. and Knoll, D. A.; "Comparison of Standard and Matrix-Free Imple- 
mentations of Several Newton-Krylov Solvers," AIAA Journal, vol. 32, no. 12, December 1994.

48. Axelsson, O.; Iterative Solution Methods, Cambridge University Press, New York, 1994, pp. 252-371.

49. Chow, E. and Saad, Y.; “Approximate Inverse Preconditioners for General Sparse Matrices," University of Minnesota Supercomputing Institute Research Report UMSI 94/ 101, May 1994.

50. Chow, E. and Saad, Y.; “Approximate Inverse Preconditioners via Sparse-Sparse Iterations," SIAM Journal of Scientific Computing, Vol. 19, No. 3, pp. 995-1023, May 1998. 51. Gould, N. I. M. and Scott, J. A.; "Sparse Approximate-Inverse Preconditioners Using Norm-Minimization Techniques," SIAM Journal of Scientific Computing, Vol. 19, No. 2, pp. 605-625, March 1998.

52. Anderson, W. K. and Bonhaus, D. L.; "An Implicit Upwind Algorithm for Computing Turbulent Flows on Unstructured Grids," Computers and Fluids, Vol. 23, No. 1, pp. 1-21, 1994.

53. Marcum, D.L. and Weatherill, N.P.; "Unstructured Grid Generation Using Iterative Point Insertion and Local Reconnection,” AIAA Journal, Vol. 33, No. 9, pp 1619-1625, September 1995.

54. Marcum, D.L.; "Unstructured Grid Generation for Viscous Flow Applications,” AIAA 33th Applied Aerodynamics Conference, AIAA 95-0212, January 1995.

55. Abbott, I. H. and von Doenhoff, A. E.; Theory of Wing Sections, Dover Publications, Inc., New York, 1959.

56. Schlichting, H.; Boundary-Layer Theory, translated by Dr. J. Kestin, seventh edition, McGraw-Hill Book Company, New York, 1979.

57. Volpe, G.; "Performance of Compressible Flow Codes at Low Mach Numbers," AIAA Journal, Vol. 31, pp. 49-56, 1993.

58. Institut National de Recherche en Informatique et en Automatique; "GAMM Workshop on Numerical Simulation of Compressible Navier-Stokes Flows,” 1985. 59. Jinyun, Y; "Symmetric Gaussian Quadrature Formulae for Tetrahedronal Regions," 
Computer Methods in Applied Mechanics and Engineering, vol. 43, 1984, pp. 349-353. 60. Schmitt, V. and Charpin, F.; "Pressure Distributions on the ONERA M6 Wing at Transonic Mach Numbers," in Experimental Data Base for Computer Program Assessment, AGARD-AR-138, pp. B1-1-B1-44, May 1979. 


\section{Appendix A}

\section{Three-Dimensional Euler Equations}

\section{A.1 Governing Equations}

The governing equations for inviscid flow in three dimensions can be written as

$$
\frac{\partial F}{\partial x}+\frac{\partial G}{\partial y}+\frac{\partial H}{\partial z}=0
$$

where

$$
Q=\left[\begin{array}{c}
\rho \\
\rho u \\
\rho v \\
\rho w \\
\rho e_{0}
\end{array}\right], F=\left[\begin{array}{c}
\rho u \\
\rho u^{2}+p \\
\rho u v \\
\rho u w \\
\rho u h_{0}
\end{array}\right], G=\left[\begin{array}{c}
\rho v \\
\rho u v \\
\rho v^{2}+p \\
\rho v w \\
\rho v h_{0}
\end{array}\right], H=\left[\begin{array}{c}
\rho w \\
\rho u w \\
\rho v w \\
\rho w^{2}+p \\
\rho w h_{0}
\end{array}\right]
$$

and $\rho$ is the density, $u, \mathrm{v}$ and $w$ are the Cartesian components of the velocity vector, $p$ is the pressure, $e$ is the internal energy per unit mass, $h$ is the enthalpy per unit mass, and a subscript " 0 " indicates a stagnation condition.

The pressure is related to density and energy via the ideal-gas equation of state given 
by

$$
p=(\gamma-1) \rho e
$$

where $\gamma$ is the ratio of specific heats.

The nondimensionalization of the equations is identical to that given in chapter 4 .

\section{A.2 Finite Element Formulation}

The SUPG weak statement corresponding to equation 100 is

$$
\int_{\Omega}\left[w+\left(\frac{\partial w}{\partial x} A+\frac{\partial w}{\partial y} B+\frac{\partial w}{\partial z} C\right) \tau\right]\left(\frac{\partial F}{\partial x}+\frac{\partial G}{\partial y}+\frac{\partial H}{\partial z}\right) d \Omega=0 .
$$

where $w$ is the Galerkin weight function, $A, B$ and $C$ are the flux Jacobian matrices corresponding to $F, G$ and $H$, and the matrix $\tau$ is defined as in chapter 2. By integrating the Galerkin terms by parts the weak statement becomes:

$$
\begin{aligned}
& \int_{\Gamma} w\left(F \hat{n}_{x}+G \hat{n}_{y}+H \hat{n}_{z}\right) d \Gamma-\int_{\Omega}\left(\frac{\partial w}{\partial x} F+\frac{\partial w}{\partial y} G+\frac{\partial w}{\partial z} H\right) d \Omega+ \\
& \int_{\Omega}\left(\frac{\partial w}{\partial x} A+\frac{\partial w}{\partial y} B+\frac{\partial w}{\partial z} C\right) \tau\left(\frac{\partial F}{\partial x}+\frac{\partial G}{\partial y}+\frac{\partial H}{\partial z}\right) d \Omega=0
\end{aligned}
$$

where the fluxes in the boundary integrals are evaluated based on the boundary conditions as described in chapter 4 .

\section{A.3 Solution Methodology}

The physical domain $\Omega$ is divided into a set of nonoverlapping tetrahedral elements

$\Omega_{e}$ such that the entire domain is represented. The solution data $Q$ are represented by tet- 
rahedral Bezier volumes in each element defined by

$$
Q(\xi, \eta, \zeta)=\sum_{i=0}^{n} \sum_{j=0}^{n-i} \sum_{k=0}^{n-i-j} Q_{i j k} B_{i j k}^{n}(\xi, \eta, \zeta)
$$

where $\xi, \eta$, and $\zeta$ are the local barycentric coordinates of the element and the $B_{i j k}^{n}$ are the trivariate Bernstein polynomials of degree $n$ given by

$$
B_{i j k}^{n}(\xi, \eta, \zeta)=\frac{n !}{i ! j ! k !(n-i-j-k) !} \xi^{i} \eta^{j} \zeta^{k}(1-\xi-\eta-\zeta)^{n-i-j-k}
$$

To accommodate curved boundaries, the coordinates of each tetrahedral element are also represented by tetrahedral Bezier volumes of degree $n$. This results in a nonlinear coordinate transformation from the physical space to the element parameter space. Derivatives in physical space $(x, y, z)$ are transformed to element parameter space $(\xi, \eta, \zeta)$ by

$$
\begin{gathered}
\frac{\partial}{\partial x}=\frac{\partial \xi}{\partial x \partial \xi}+\frac{\partial \eta}{\partial x} \frac{\partial}{\partial \eta}+\frac{\partial \zeta \partial}{\partial x \partial \zeta}, \frac{\partial}{\partial y}=\frac{\partial \xi}{\partial y \partial \xi}+\frac{\partial \eta}{\partial y} \frac{\partial}{\partial \eta}+\frac{\partial \zeta \partial}{\partial y \partial \zeta}, \\
\frac{\partial}{\partial z}=\frac{\partial \xi}{\partial z} \frac{\partial}{\partial \xi}+\frac{\partial \eta}{\partial z} \frac{\partial}{\partial \eta}+\frac{\partial \zeta}{\partial z} \frac{\partial}{\partial \zeta}
\end{gathered}
$$

The metric terms appearing in equation 107 can be expressed in terms of the derivatives of the element coordinates as

$$
\begin{aligned}
& \frac{\partial \xi}{\partial x}=\frac{1}{J}\left(\frac{\partial y \partial z}{\partial \eta \partial \zeta}-\frac{\partial z \partial y}{\partial \eta \partial \zeta}\right) \frac{\partial \xi}{\partial y}=\frac{1}{J}\left(\frac{\partial z \partial x}{\partial \eta \partial \zeta}-\frac{\partial x \partial z}{\partial \eta \partial \zeta}\right) \frac{\partial \xi}{\partial z}=\frac{1}{J}\left(\frac{\partial x \partial y}{\partial \eta \partial \zeta}-\frac{\partial y \partial x}{\partial \eta \partial \zeta}\right) \\
& \frac{\partial \eta}{\partial x}=\frac{1}{J}\left(\frac{\partial z \partial y}{\partial \xi \partial \zeta}-\frac{\partial y \partial z}{\partial \xi \partial \zeta}\right) \frac{\partial \eta}{\partial y}=\frac{1}{J}\left(\frac{\partial x \partial z}{\partial \xi \partial \zeta}-\frac{\partial z \partial x}{\partial \xi \partial \zeta}\right) \frac{\partial \eta}{\partial y}=\frac{1}{J}\left(\frac{\partial y \partial x}{\partial \xi \partial \zeta}-\frac{\partial x \partial y}{\partial \xi \partial \zeta}\right) \\
& \frac{\partial \zeta}{\partial z}=\frac{1}{J}\left(\frac{\partial y \partial z}{\partial \xi \partial \eta}-\frac{\partial z \partial y}{\partial \xi \partial \eta}\right) \frac{\partial \zeta}{\partial z}=\frac{1}{J}\left(\frac{\partial z \partial x}{\partial \xi \partial \eta}-\frac{\partial x \partial z}{\partial \xi \partial \eta}\right) \frac{\partial \zeta}{\partial z}=\frac{1}{J}\left(\frac{\partial x \partial y}{\partial \xi \partial \eta}-\frac{\partial y \partial x}{\partial \xi \partial \eta}\right)
\end{aligned}
$$

where $J$ is the Jacobian of the element coordinate transformation defined by

$$
J=\frac{\partial x \partial y \partial z}{\partial \xi \partial \eta \partial \zeta}+\frac{\partial y \partial z \partial x}{\partial \xi \partial \eta \partial \zeta}+\frac{\partial z \partial x \partial y}{\partial \xi \partial \eta \partial \zeta}-\frac{\partial x \partial z \partial y}{\partial \xi \partial \eta \partial \zeta}-\frac{\partial y \partial x}{\partial \xi \partial \eta \partial \zeta}-\frac{\partial z \partial y \partial x}{\partial \xi \partial \eta \partial \zeta}
$$

Finally, the volume differential $d \Omega_{e}$ is scaled by the Jacobian $J$ as follows: 


$$
d \Omega_{e}=J d \xi d \eta d \zeta
$$

Continuity of the solution across element interfaces is enforced by sharing control points along the interfaces as illustrated in figure 23a. The $(i, j, k)$ indexing in equation 105 is converted to a single index as shown in figure $23 \mathrm{~b}$ by the following function:

$$
i_{\text {node }}=k\left(3 n^{2}+12 n+11-3 n k-6 k+k^{2}\right) / 6+j(2 n-2 k-j+3) / 2+i
$$

The boundary $\Gamma$ of the domain $\Omega$ is divided into a finite number of triangular elements $\Gamma_{e}$, each of which corresponds to the face of a tetrahedral element adjoining the boundary. The coordinates and data on each of these elements is represented by a triangular Bezier patch of degree $n$ as described in chapter 4 . Integrals over the boundary $\Gamma$ can now be written as sums of integrals over individual boundary elements. These element integrals are transformed into integrals over the local element parameter space.

The polynomial expression of the boundary element coordinates gives rise to a continuously varying unit normal along curved elements. The components of the element normal vector $n_{x}, n_{y}$ and $n_{z}$ are given by

$$
n_{x}=\frac{\partial z \partial y}{\partial \xi \partial \eta}-\frac{\partial y \partial z}{\partial \xi \partial \eta}, n_{y}=\frac{\partial x \partial z}{\partial \xi \partial \eta}-\frac{\partial z \partial x}{\partial \xi \partial \eta}, n_{z}=\frac{\partial y \partial x}{\partial \xi \partial \eta}-\frac{\partial x \partial y}{\partial \xi \partial \eta}
$$

Boundary element integrals are transformed according to

$$
\int_{\Gamma_{e}} f(x, y, z) d \Gamma_{e}=\int_{0}^{11} \int_{0}^{1-\eta} f[x(\xi, \eta), y(\xi, \eta), z(\xi, \eta)] R d \xi d \eta
$$

where

$$
R=\sqrt{\left(\frac{\partial z \partial y}{\partial \xi \partial \eta}-\frac{\partial y \partial z}{\partial \xi \partial \eta}\right)^{2}+\left(\frac{\partial x \partial z}{\partial \xi \partial \eta}-\frac{\partial z \partial x}{\partial \xi \partial \eta}\right)^{2}+\left(\frac{\partial y \partial x}{\partial \xi \partial \eta}-\frac{\partial x \partial y}{\partial \xi \partial \eta}\right)^{2}}
$$

Upon substitution of the foregoing domain and boundary transformations, the weak statement (equation 104) can now be written as 


$$
\begin{aligned}
& \sum_{\Gamma_{e}}\left[\int_{0}^{1} \int_{0}^{1-\eta} w\left(F \hat{n}_{x}+G \hat{n}_{y}+H \hat{n}_{z}\right) R d \xi d \eta\right]+ \\
& \sum_{\Omega_{e}}\left[-\int_{0}^{11} \int_{0}^{0} \int_{0}^{0}\left(\frac{\partial w}{\partial \xi} \tilde{F}+\frac{\partial w}{\partial \eta} \tilde{G}+\frac{\partial w}{\partial \zeta} \tilde{H}\right) J d \xi d \eta d \zeta\right. \\
& \left.+\int_{0}^{11} \int_{0}^{1-\eta} \int_{0}^{-\zeta}\left(\frac{\partial w}{\partial \xi} \tilde{A}+\frac{\partial w}{\partial \eta} \tilde{B}+\frac{\partial w}{\partial \zeta} \tilde{C}\right) \tau\left(\tilde{A} \frac{\partial Q}{\partial \xi}+\tilde{B} \frac{\partial Q}{\partial \eta}+\tilde{C} \frac{\partial Q}{\partial \zeta}\right) J d \xi d \eta d \zeta\right]=0
\end{aligned}
$$

where

$$
\tilde{F}=\frac{\partial \xi}{\partial x} F+\frac{\partial \xi}{\partial y} G+\frac{\partial \xi}{\partial z} H, \tilde{G}=\frac{\partial \eta}{\partial x} F+\frac{\partial \eta}{\partial y} G+\frac{\partial \eta}{\partial z} H, \tilde{G}=\frac{\partial \zeta}{\partial x} F+\frac{\partial \zeta}{\partial y} G+\frac{\partial \zeta}{\partial z} H
$$

are the transformed inviscid flux vectors and $\tilde{A}, \tilde{B}$ and $\tilde{C}$ are the corresponding flux Jacobians.

The integrals appearing in equation 115 are evaluated numerically using the Gaussian quadrature rules of [59] for tetrahedral elements and [44] for the triangular elements on the boundary. Availability of quadrature rules for tetrahedral and triangular elements limits the scheme to fifth order accuracy.

The solution $Q$ is obtained using a Newton-Krylov scheme as described in chapter 4. The only difference is the preconditioner, which is still of block-diagonal form and is given by

$$
\begin{aligned}
& \frac{\partial R_{m}}{\partial Q_{m}} \approx \sum_{\Gamma_{e}}\left[\int_{0}^{11-\eta} \int_{0}^{n} B_{i j k}^{n}\left(A \hat{n}_{x}+B \hat{n}_{y}+C \hat{n}_{z}\right) B_{i j k}^{n} R d \xi d \eta\right]+
\end{aligned}
$$

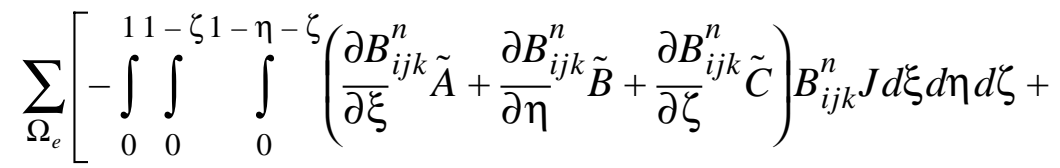

$$
\begin{aligned}
& \left.\int_{0}^{11} \int_{0}^{-\zeta 1-\eta} \int_{0}^{-\zeta}\left(\frac{\partial B_{i j k}^{n}}{\partial \xi}{ }^{n}+\frac{\partial B}{\partial \eta}_{i j k}^{n} \tilde{B}+\frac{\partial B_{i j k}^{n}}{\partial \zeta} \tilde{C}\right) \tau\left(\tilde{A} \frac{\partial B_{i j k}^{n}}{\partial \xi}+\tilde{B} \frac{\partial B}{\partial \eta}_{i j k}^{n}+\tilde{C}{\frac{\partial B_{i j k}^{n}}{\partial \zeta}}^{n}\right) J d \xi d \eta d \zeta\right]
\end{aligned}
$$




\section{A.4 Results}

\subsubsection{NACA 0012 Wing}

The geometry consists of a straight wing with a constant NACA 0012 cross section between two vertical walls. To produce grids for higher order solutions, a least-squares procedure similar to that used in two dimensions is used to approximate the true surfaces. The three corners of each surface triangle match the true surface exactly.

Figure 24 shows mid-span pressure distributions at a Mach number of 0.63 and an angle of attack of 2 degrees. Note that the third order solution has a much higher suction peak and is comparable to the second order solution using a more refined grid.

\subsubsection{ONERA M6 Wing}

Subcritical flow over the ONERA M6 wing at a Mach number of 0.699 and an angle of attack of 3.06 degrees was computed using the 2 nd order scheme. The geometry and experimental data for this case are discussed in reference 60. Figure 25 shows the pressure distribution at $65 \%$ span compared with experimental data. The comparison is favorable even though the grid is relatively coarse. Higher order solutions have not yet been obtained due to the difficulty in obtaining higher order representations of the surface. 


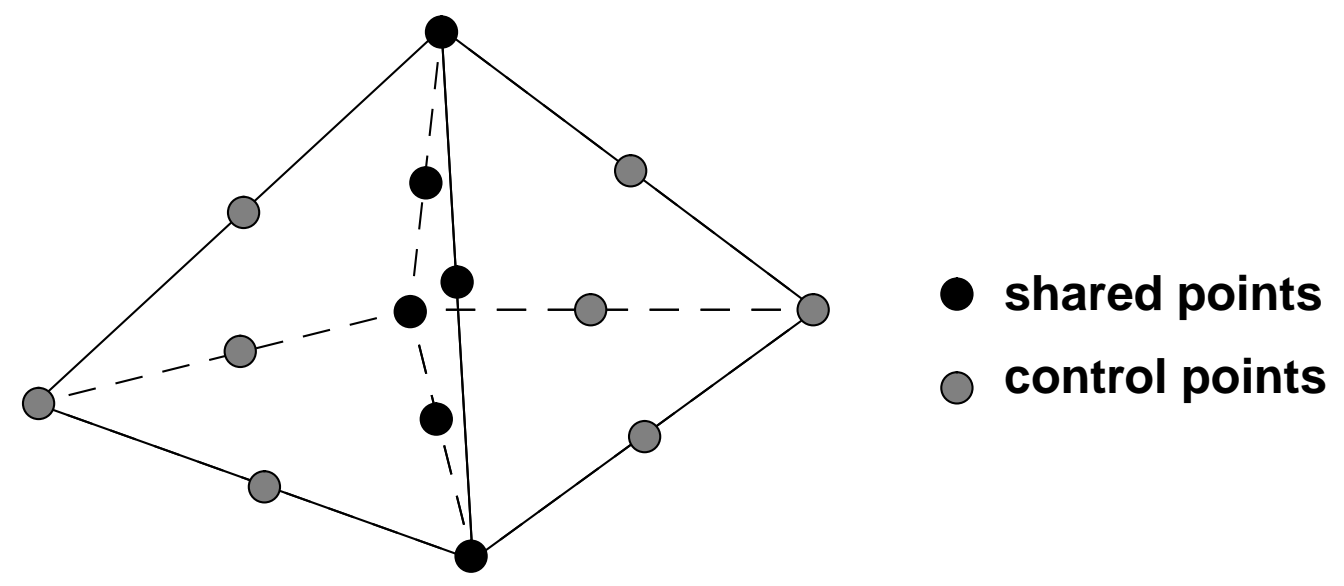

a) Enforcement of continuity via shared control points (quadratic elements depicted).

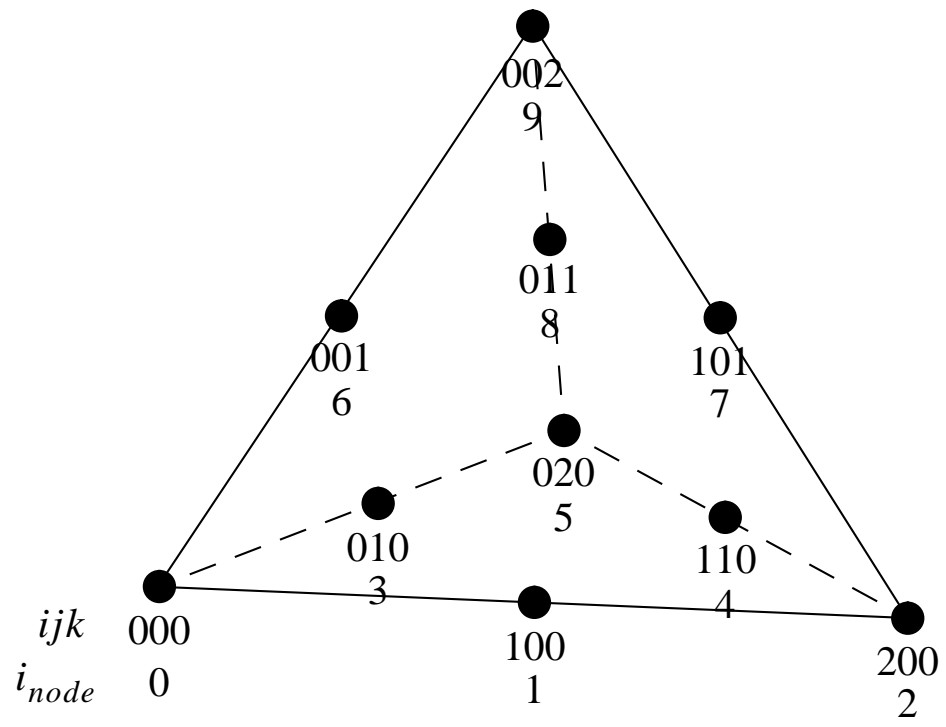

b) Indexing of control points within an element (quadratic element depicted).

Figure 23. Element data distribution using triangular Bezier patches. 


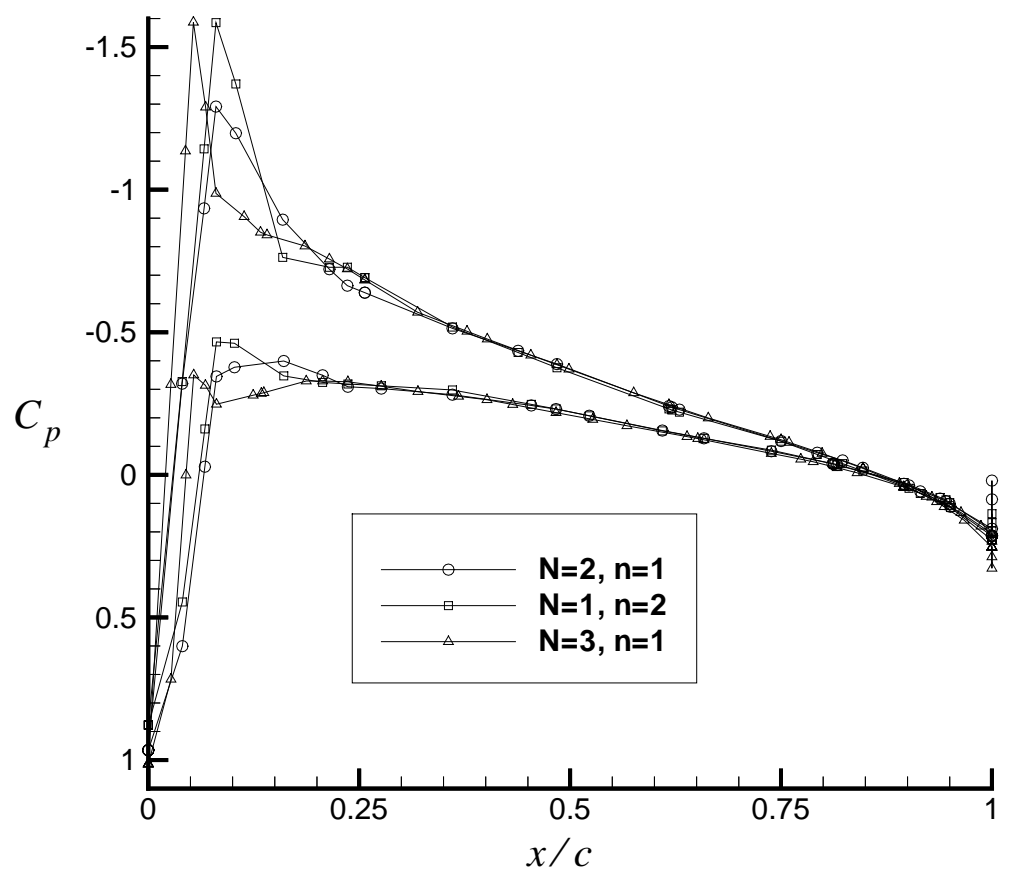

Figure 24. Mid-span surface pressure distribution for NACA 0012 wing. 


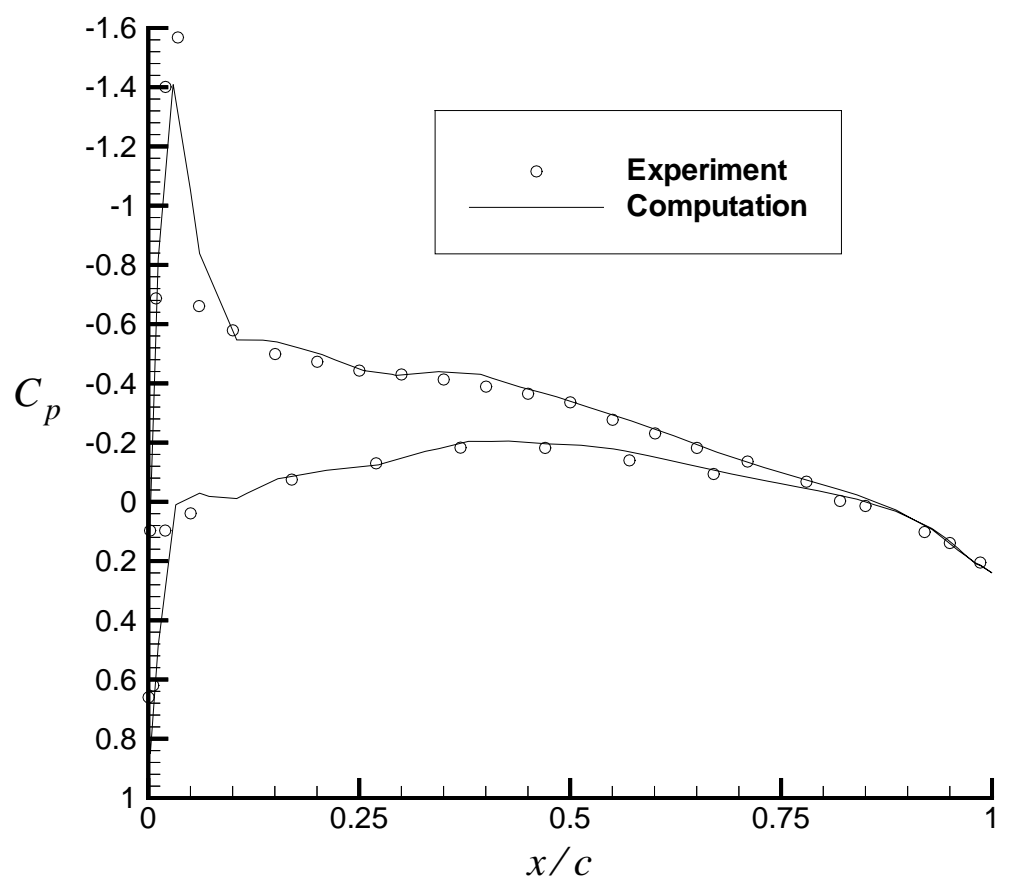

Figure 25. Surface pressure distribution for ONERA M6 wing at 65\% span. 


\section{Vita}

Daryl L. Bonhaus was born December 11, 1967 in Cincinnati, Ohio. He graduated from Elder High School in Cincinnati in June of 1985 and received a B.S. in Aerospace Engineering in June of 1990 at the University of Cincinnati. Upon graduation, he began working at the Langley Research Center of the National Aeronautics and Space Administration in Hampton, Virginia. He attained a M.S. in Fluid Mechanics and Thermal Sciences from the George Washington University in September of 1993. Daryl continues to work for the Langley Research Center. 